\title{
PALEO
}

Revue d'archéologie préhistorique

\section{La place et les caractéristiques du débitage sur enclume (" bipolaire ») dans les industries brésiliennes}

Place and characteristics of bipolar-on-anvil debitage in Brazilian industries

André Prous, Márcio Alonso, Gustavo Neves de Souza, Angelo Pessoa Lima et Filipe Amoreli

\section{OpenEdition}

Journals

Édition électronique

URL : http://journals.openedition.org/paleo/1996

DOI : $10.4000 /$ paleo.1996

ISSN : 2101-0420

Éditeur

SAMRA

Édition imprimée

Pagination : 201-219

ISSN : 1145-3370

Référence électronique

André Prous, Márcio Alonso, Gustavo Neves de Souza, Angelo Pessoa Lima et Filipe Amoreli, «La place et les caractéristiques du débitage sur enclume ("bipolaire ») dans les industries brésiliennes », PALEO [En ligne], Numéro spécial | 2009-2010, mis en ligne le 23 avril 2012, consulté le 07 juillet 2020 URL : http://journals.openedition.org/paleo/1996 ; DOI : https://doi.org/10.4000/paleo.1996 


\title{
LA PLACE ET LES CARACTÉRISTIQUES DU DÉBITAGE SUR ENCLUME («BIPOLAIRE ») DANS LES INDUSTRIES BRÉSILIENNES
}

\author{
André PROUS ${ }^{(1)}$, Márcio ALONSO (1), Gustavo NEVES de SOUZA ${ }^{(1)}$, \\ Angelo PESSOA LIMA ${ }^{(1)}$ et Filipe AMORELI ${ }^{(1)}$
}

\begin{abstract}
Résumé : Les industries taillées sur enclumes sont très abondantes au Brésil et dans le nord-est de l'Uruguay. Cette technique domine particulièrement dans les industries sur quartz (région de Lagoa Santa depuis le Pléistocène final ; sites mésolithiques coquilliers du littoral méridional ; horticulteurs Tupiguarani), sur agate (extrême sud du Brésil) voire sur basalte dans certaines régions (Pantanal du Mato Grosso) mais intéresse aussi certaines pièces de silex ou de quartzite, même dans les ensembles où domine la taille à main libre. À partir de nos expériences de fabrication et de l'observation de nombreux ensembles archéologiques, nous exposons les caractéristiques de la taille sur enclume sur les éclats, les percuteurs et les enclumes, ainsi que certaines causes d'erreur de diagnostic relevées dans la bibliographie. Il est en effet possible de différencier les enclumes et percuteurs utilisés pour la taille de ceux qui ont servi au broyage de pigments ou à casser des noix de palmes et autres végétaux durs. On montre que la taille sur enclume n'est pas seulement une technique utilisée par de mauvais tailleurs ou quand on ne dispose pas de matières premières de qualité, de forme ou de dimensions adéquates : elle est également très rentable et efficace, permettant d'obtenir presque tous les principaux types de tranchants et de pointes sans nécessité de retouche. Cette utilisation préférentielle de la taille sur enclume par des populations qui dominaient d'autres techniques est illustrée par les industries du Brésil central (Lagoa Santa, Serra do Cipó, Diamantina dans l'état de Minas Gerais), constituées pour l'essentiel d'éclats obtenus sur enclume, mais qui comportent au début de l'Holocène de très beaux grattoirs retouchés et de magnifiques pointes de trait bifaciales en quartz. Le marteau et l'enclume ont été utilisés non seulement pour l'extraction d'éclats, mais aussi pour la préparation de préformes de lames de hache en quartzite ou diabase (façonnage appuyé sur enclume) et pour la fabrication - encore pratiquée en Amazonie sur le haut Rio Negro - de dents de râpes à manioc (percussion sur enclume de quartz et de diabase). Nous concluons que la taille à main libre - suivie ou non de la pratique de la retouche - n'est pas indispensable et que la recherche de produits standardisés par des méthodes de taille spécifiques à main libre et des retouches systématiques illustrent un choix culturel et non une amélioration technique en soi. Les produits du débitage sur enclume peuvent parfaitement répondre à la plupart des nécessités remplies par des outils de pierre.
\end{abstract}

Mots-clés : Brésil, technologie lithique, débitage sur enclume, façonnage sur enclume, enclumes, percuteurs, industries de quartz.

Abstract: Place and characteristics of bipolar-on -anvil debitage in Brazilian industries. The bipolar technique is very common in Brazil and in northeastern Uruguay. It is the primary technique used, especially where most chipped lithic instruments are made of quartz (as in the Lagoa Santa region, dating from the late Pleistocene, in mesolithic shell middens of the coastal range, among Tupiguarani horticulturalists), of agate (as found in the extreme south of Brazil), or even of basalt, as is the case in some regions (for example, in the marshes of the area of the Pantanal in Mato Grosso). Even in the regions where free-hand reduction is dominant, some flint or quartzite instruments, made by way of the bipolar technique, can be found. On the basis of our experiments, and based on the observation of many archaeological collections, we describe the peculiarities of the marks left by bipolar flaking on anvil stones, on hammer stones, and on the flakes. It is possible to differentiate the instruments used to flake stone on the anvil from the instruments used to crush minerals (pigments) or other hard materials like coconuts. We think that bipolar flaking is not restricted to unskilled people or those who had no good raw materials on hand (stones of bad quality or of an inadequate shape or dimension). In fact, this technique is very efficient and productive, allowing for the generation of almost every kind of cutting or drilling edge, with no need of retouching. The dominant use of anvil techniques by populations that also knew how to utilize other, more sophisticated flaking and retouch techniques is evident in central Brazil (Minas Gerais) where mesolithic people were able to make delicate end scrapers and magnificent bifacial projectile points on crystalline quartz, but mainly used bipolar unretouched flakes. Flaking on anvil stone has been used not only for core reduction but also for the preparation of basalt or quartzite axe preforms. In Amazonia (Rio Negro Valley), Baniwa and Desana Indians are making basalt or quartz teeth for manioc rasps from anvil stone even today. We conclude that free hand flaking is not a necessary technique to obtain good stone cutting edges. So, the custom of using complex flaking methods and the systematic retouching of instruments during Palaeolithic times in some parts of the World was more a cultural choice than a technical improvement.

Key-words: Brazil, lithic Technology, bipolar technique, anvil stones, hammerstones, quartz industries. 


\section{INTRODUCTION}

Au début des années 1980, nous avons pu vérifier, avec J. Flenniken, que les industries lithiques de la fameuse région archéologique de Lagoa Santa avaient été produites sur enclume. À partir de ce moment, nous avons procédé à de nombreuses expériences qui ont contribué à vérifier les caractéristiques des restes de débitage (Prous et Alonso 1986) - jusque là souvent mal comprises par les chercheurs (p. ex. : Kobayashi 1975 et Miller 1975 et 1979) ; surtout, nous avons pu montrer les particularités des instruments de taille percuteurs et enclumes - qui avaient été jusqu'alors délaissés (Moura et Prous 1989). Par la suite, nous avons été amenés à identifier l'utilisation du débitage sur enclume dans d'autres industries du Brésil, ainsi que d'Uruguay, d'Amérique centrale, d'Australie, d'Italie et d'Espagne.

L'utilisation de l'enclume pour la fabrication d'outils ne se limite d'ailleurs pas au travail de la pierre et nous l'avons observée et reproduite expérimentalement pour le travail des coquilles au Brésil.

L'emploi d'enclumes de pierre brute est traditionnel au Brésil, où l'on trouve encore dans les fermes, au pied des palmiers, des blocs de pierre destinés au bris des noix de palme natives (beaucoup plus petites que les noix de coco d'origine africaine consommées en France). Le couple enclume-percuteur pour casser les noix, mentionné au début du $\mathrm{XIX}^{\mathrm{e}}$ siècle par le naturaliste J. M. Rugendas (1835) comme un instrument omniprésent dans les habitations indigènes, pourrait même être antérieur à l'arrivée de l'Homme sur le continent américain. En effet, nous avions observé, en 1971, des singes du genre Cebus cassant des noix entre deux pierres dans le Jardin zoologique de Rio de Janeiro. Nous nous demandions si cela ne serait pas une conduite influencée par la proximité des traiteurs ; mais notre collègue $\mathrm{E}$. Fogaça (communication personnelle, faite il y a une dizaine d'années) recueillit auprès de paysans de l'état de Goiás une information selon laquelle des singes à l état sauvage procèderaient de même aux alentours des fermes isolées.

Quoi qu'il en soit, les enclumes sont extrêmement nombreuses dans les sites archéologiques du Brésil, où elles étaient traditionnellement identifiées comme des cassenoix (quebra cocos). Passer du bris des noix à celui des pierres est une opération très simple qui put se réaliser il y a fort longtemps.

Lors d'une présentation de nos recherches, faite au laboratoire du CNRS «Préhistoire et Technologie » à Meudon en 1985, Hélène Roche nous montra des éclats et fragments en provenance d'Éthiopie, associés à des sites du Paléolithique archaïque ; ils avaient évidemment été produits sur enclume et nous sommes persuadés que cette technique dut être découverte par les hominidés dès l'aurore de la taille de la pierre, peut-être même avant la percussion à main libre.
Traditionnellement dédaigné, trop souvent ignoré par les chercheurs, nous croyons que le débitage sur enclume fut un des modes de travail les plus répandus au cours de toute la préhistoire dans une bonne partie du monde. Non par faute de connaissance d'autres techniques ou lorsqu'on ne disposait pas de matières premières de qualité suffisante pour contrôler la taille, mais surtout parce qu'il s'agit d'une technique extrêmement efficace et qui permet d'obtenir facilement presque tous les types de tranchants et parties actives désirables.

Nous présenterons d'abord l'extension des territoires brésiliens dans lesquels les industries lithiques privilégient le débitage sur enclume et expliquerons les raisons de ce choix. Ensuite, nous montrerons les formes reconnues dans les collections, leur mode d'obtention - à partir de nos expériences - et les difficultés pour identifier l'origine de certains éclats. Nous verrons ensuite les différences que présentent les produits du travail sur enclume en fonction des matières premières travaillées et des objectifs des tailleurs. Une partie spéciale exposera nos premières expériences en vue de tester la fonction ou l'origine de certaines pièces esquillées. Finalement, nous montrerons la différence entre les stigmates laissés sur le couple enclumepercuteur par le débitage de la pierre ou des pointes et ceux qui proviennent du bris de produits végétaux.

Notons que, dorénavant, nous désignerons par l'expression « taille bipolaire » (déjà utilisée par Breuil et Lantier 1951) ce que les chercheurs formés par J. Tixier appellent « taille sur enclume ».

\section{1 - LES INDUSTRIES LITHIQUES BRÉSILIENNES À DÉBITAGE BIPOLAIRE (= sur enclume) (fig. 1)}

- Le premier ensemble régional correspond à une bonne partie de l'État de Minas Gerais (Brésil central) - premier producteur mondial de cristal de quartz monocristallin. Dans les régions cristallines, le silex est souvent absent et le quartz est la meilleure roche disponible qui permette de créer des pointes ou des tranchants très aigus et ne s'émoussant pas trop rapidement. Les outils qui exigent un tranchant plus robuste sont faits sur des plaquettes de quartzite (Serra do Cipó, près de Lagoa Santa). Le quartz, seul, est travaillé sur enclume. L'outillage lié au débitage sur enclume comporte, outre le quartz comme matière à débiter, des percuteurs et des enclumes en roches ultra basiques (Lagoa Santa), en tillites (Serra do Cipó) ou en granite (vallée du Rio Doce).

- Le second ensemble correspond au littoral central et méridional du Brésil, à la vallée du rio Doce ainsi qu'à la côte nord de l'Uruguay. Cette région côtière offre des filons de quartz intrusifs dans les roches cristallines et des dykes de basalte et de diabase ; au sud, les druses d'agate sont nombreuses dans les coulées basaltiques. Bien que les basaltes se taillent - assez mal, à vrai dire - ce sont les quartz et les agates qui fournissent généralement les tranchants aigus et les pointes de projectile, presque exclusivement à partir d'un travail bipolaire. 


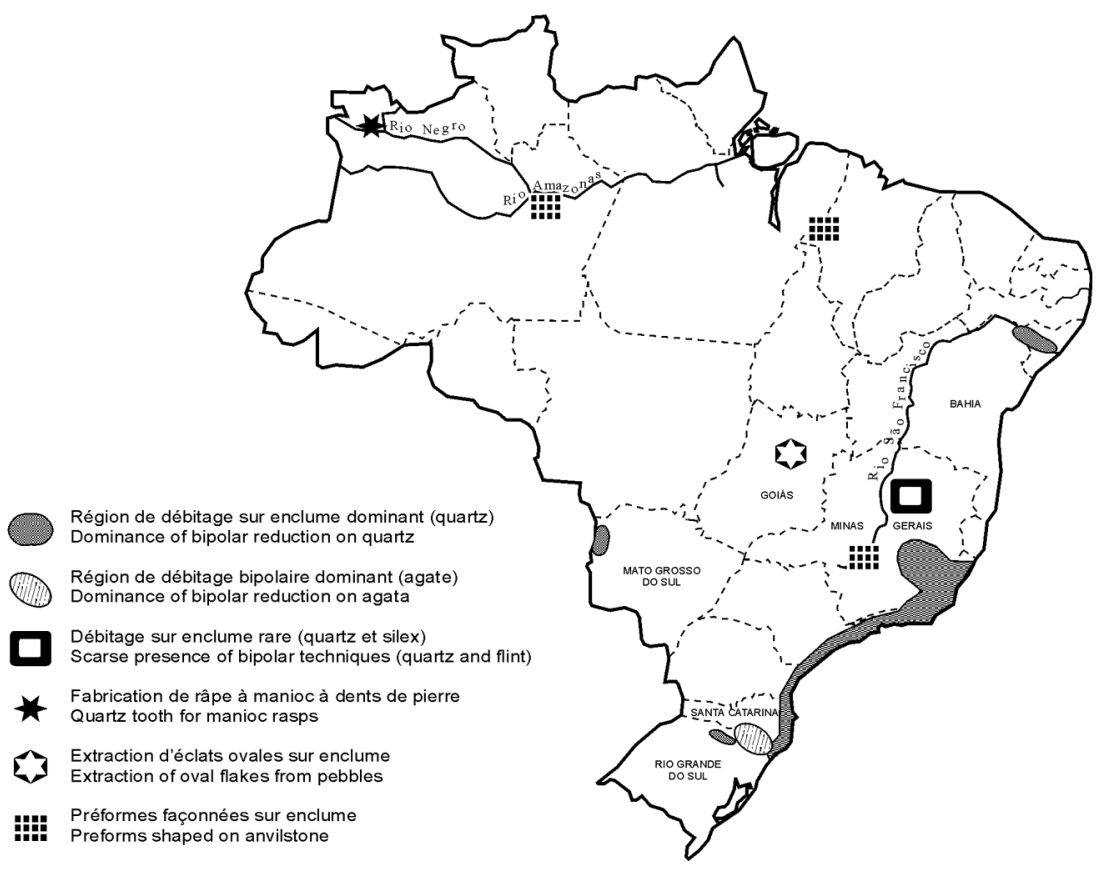

Figure 1 - Le travail sur enclume de la pierre au Brésil.

Figure 1 - The use of bipolar technique in Brazil.

a

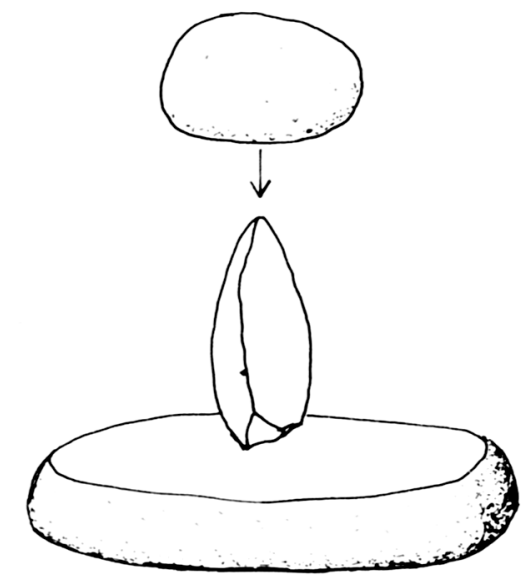

Figure 2 - La fracture des roches sur enclume.

a : position du bloc à débiter et direction des coups portés ;

$b$ : cicatrice des coups de stabilisation du bloc;

$c$ : fente;

$d$ : produits principaux

(1: éclats bipolaires marginaux, au talon parfois non écrasé ; 2 : pièce nucléiforme ; 3 : éclat bipolaire typique

b
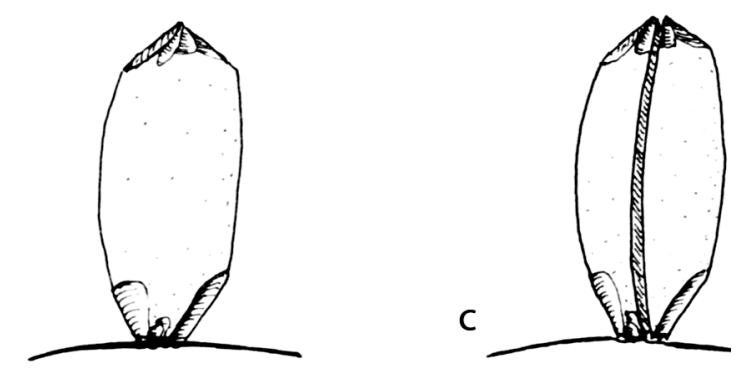
avec marques de contre-coups).

Figure 2 - Reduction of bipolar core :

a : position of the core and direction of the blows ;

$b$ : stabilization scars ;

$c$ : splitting;

$d$ : products of bipolar reduction (1: marginal bipolar flakes ; 2 : bipolar core (nucléiforme) ; 3 : complete bipolar flake with counter-blow scars).

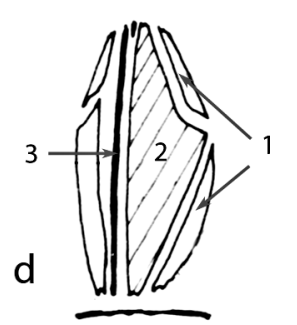


- Dans le Pantanal du Mato Grosso du sud, la taille semble avoir été exclusivement sur enclume et a été appliquée aux matériaux les plus divers : au quartz, bien sûr, mais aussi aux quartzites, à la calcédoine, à l'hématite compacte et même aux schistes, au calcaire et aux siltites (Schmitz et al. 1998).

- Partout ailleurs à l'intérieur des terres, là où abondent les silex et les quartzites d'excellente qualité (dits " grès silicifiés " dans la nomenclature américaine) on trouve des industries taillées essentiellement à main libre. On constate cependant par endroits l'utilisation épisodique de la taille sur enclume, dans les mêmes ensembles industriels dominés par la percussion directe (cours moyen du Rio São Francisco, par exemple). Ce sont alors aussi bien des objets de quartz (minoritaire) que de silex ou de quartzite.

- En Amazonie, les industries lithiques sont encore très peu étudiées mais S. Rostain (1994) a pu vérifier l'existence de la taille bipolaire sur enclume sur les quartz de Guyane, tandis que nous l'avons identifiée sur les quartzites de la région de Manaus étudiés par F. Costa. Signalons enfin que les indiennes Baniwa du haut Rio Negro concassent encore la pierre pour obtenir des dents de racloir à manioc.

On voit donc que les industries sur quartz - cristallin ou de filon - ou sur agate sont particulièrement liées au débitage sur enclume, à toutes les époques de la préhistoire locale, alors que cette technique n'a été utilisée qu'occasionnellement pour le silex, le quartzite, voire pour des roches tenaces.

\section{2 - LA TECHNIQUE ET LES PRODUITS DE LA TAILLE BIPOLAIRE}

\section{Le débitage (fig. 2)}

V. Mourre (1996) a écrit que la percussion sur enclume permet " d'obtenir des séries d'éclats fonctionnels de formes [statistiquement] prévisibles » (le mot « statistiquement » est de nous), à partir de blocs défavorables en raison de leur matière première (quartz), de la forme des blocs (galets ovoïdes, blocs massifs ne présentant pas d'angles pour l'attaque) ou de leurs faibles dimensions. Outre le fait qu'elle est extrêmement efficace, elle a l'avantage d'être très facile à appliquer.

II ne s'agit pourtant pas toujours d'un pis-aller, mais parfois d'un choix volontaire pour obtenir quelques-unes des formes spécifiques produites par cette technique. On voit en effet de très bons silex, voire des obsidiennes (nous l'avons identifiée pour la première fois dans le Panamá dans cette matière, sur une série où elle était la technique dominante) et des andésites débités de cette façon. Bien des blocs de forme massive fendus initialement sur enclume pourraient être exploités ensuite à main libre, mais continuent à être travaillés par percussion bipolaire, même dans des groupes qui connaissent la percussion libre.

Par ailleurs, la percussion sur enclume permet d'exploiter des blocs ou des galets de dimensions très restreintes (moins de $2 \mathrm{~cm}$ ), puisque la limite est seulement l'épaisseur des doigts qui les maintiennent (fig. 3). De plus, les éclats

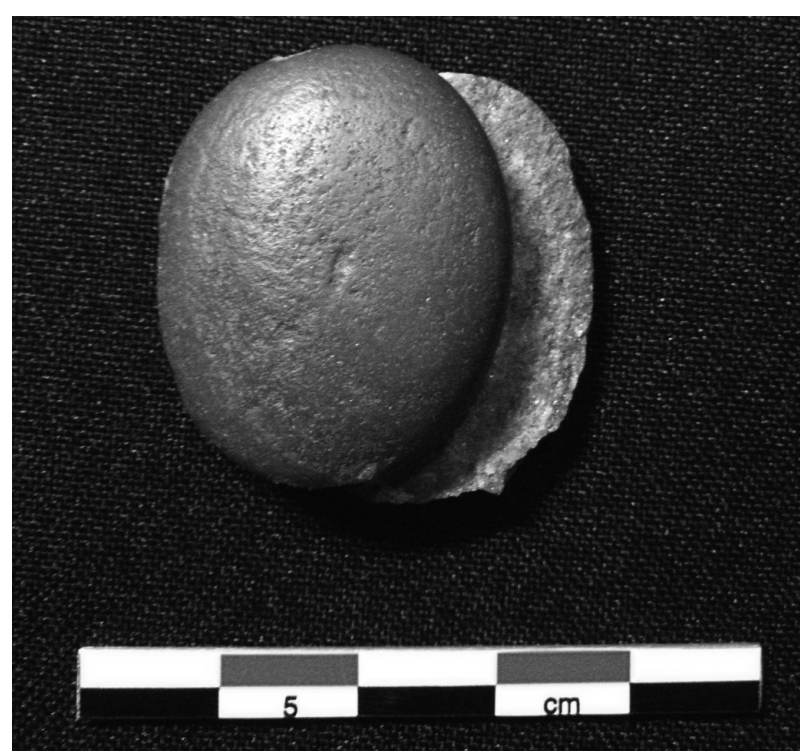

Figure 3 - Hémilite expérimental sur petit galet de grès.

Figure 3 - Experimental hemilite on a little sandstone pebble.

pouvant traverser toute la pièce nucléiforme et ce, sans risquer un phénomène d'outrepassage, on peut exploiter au maximum la dimension du bloc travaillé. Finalement, l'absence de bulbe ou de contrebulbe sur la presque totalité des produits permet d'obtenir des éclats très minces et de profil rectiligne, particulièrement adaptés à l'emmanchement.

En effet, l'extraction des éclats se fait beaucoup plus par fracture plane que selon un système d'ondes hertziennes ; cela fait que la fracture est rarement conchoïdale, même sur les roches fragiles et que cette technique est applicable aux roches tenaces. Cette caractéristique explique pourquoi beaucoup de chercheurs ne savent pas «voir » les produits de cette forme de débitage qui ne présentent souvent aucun des caractères classiques décrits par les préhistoriens depuis le $\mathrm{XIX}^{\mathrm{e}}$ siècle (talon, bulbe, voire ondes). Ce ne sont pas seulement ces particularités des éclats et des pièces de rebut (lesquelles ne ressemblent pas non plus à des nucléus classiques puisqu'elles ne présentent pas de plan de frappe) qui rendent cette méthode incompréhensible pour qui est habitué aux industries de débitage à main libre (unipolaire dans son acception originale) : la procédure mentale est inverse.

Dans le cas du débitage unipolaire, on a la démarche présentée à la figure 4. Dans le cas du débitage bipolaire, on a la démarche présentée à la figure 5 .

L'objet à débiter est placé sur une enclume à surface plane (fig. 6, 7, 8) ; il est maintenu verticalement, entre le pouce et l'index et disposé de manière à présenter au choc une arête ou un point, jamais une surface. On le frappe avec un percuteur lourd, souvent légèrement aplati ; le percuteur attaque perpendiculairement à l'enclume, frappant par une de ses faces (pour permettre de frapper avec le maximum de force et sans risquer d'abîmer la main qui tient le bloc). 


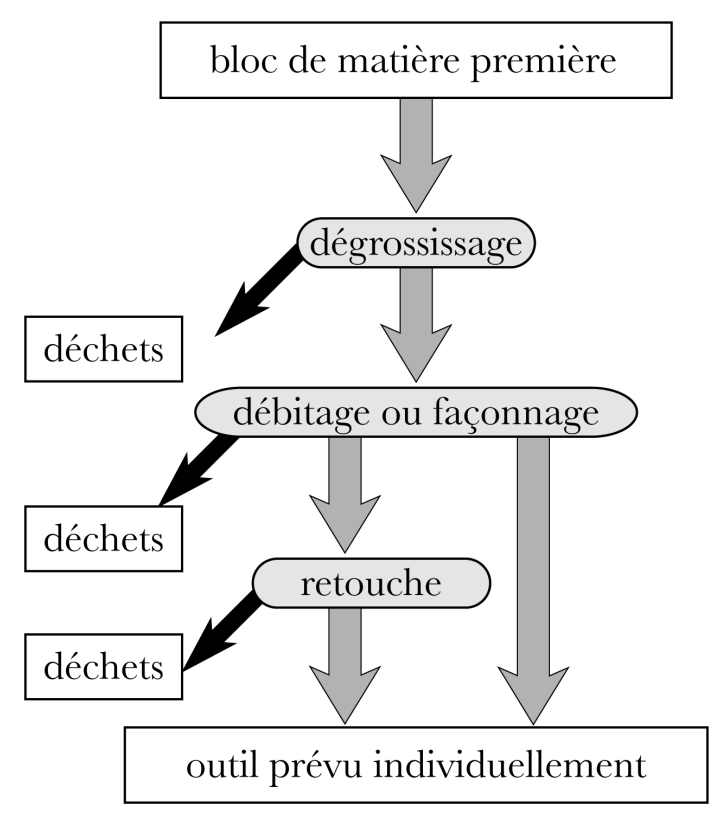

Figure 4 - Représentation schématique de la démarche de la percussion « unipolaire ».

Figure 4 - Schematic representation of the « unipolar » free-hand flaking.

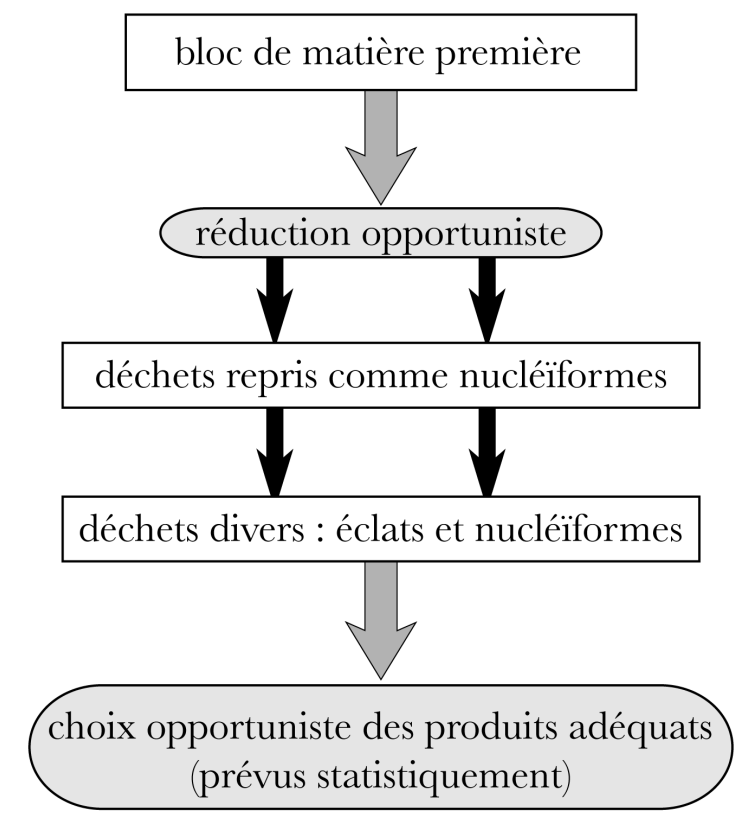

Figure 5 - Représentation schématique de la démarche de la percussion « bipolaire » sur enclume.

Figure 5 - Schematic representation of the « bipolar » on anvil flaking.

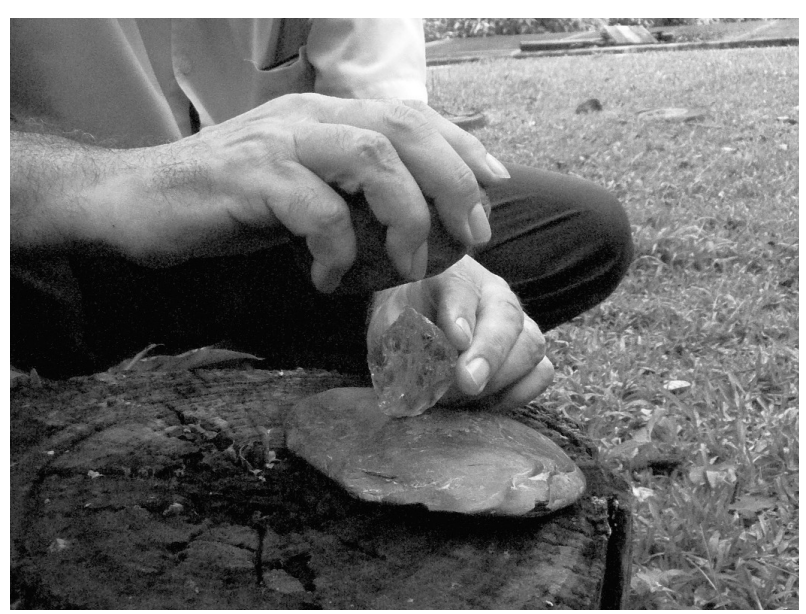

Figure 6 - Position des outils et du bloc à débiter.

Figure 6 - Position of anvilstone, hammerstone and core.

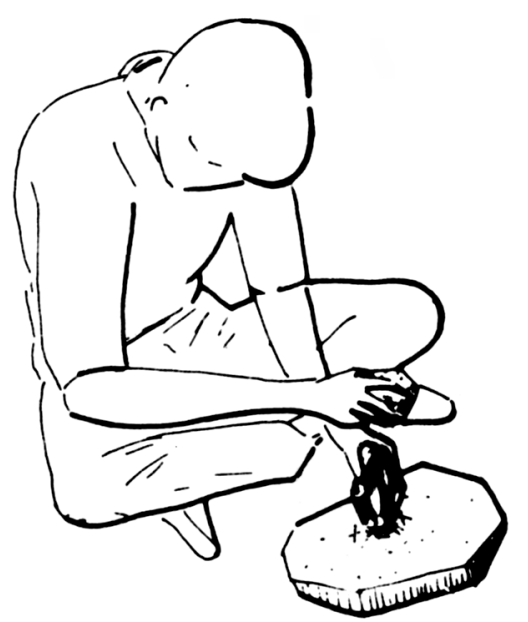

Figure 7 - Position du corps lors du débitage.

Figure 7 - Position of the body during the reduction process.

Quelques coups légers permettent d'abord d'“ asseoir " l'objet par le retrait de petites esquilles qui régularisent sa base, puis des coups violents permettent de le fendre ; chaque partie détachée devient un nouveau bloc à débiter. II n'y a donc pas de différence réelle entre nucléus et éclats, mais entre ce que nous avons appelé des pièces nucléiformes (qui peuvent encore être débitées) et des produits plus minces - que nous appelons éclats bipolaires (fig. 9 et annexe). La différence n'est pas dans la présence d'une face interne (avec bulbe) opposée à une face externe (qui déterminerait les éclats) ou d'un bloc avec des contrebulbes (identifiant un nucléus). En effet, hormis les parties externes corticales, tous les produits (nucléiformes inclus) sont délimités par deux, trois ou quatre faces d'éclatement, d'où les bulbes sont généralement absents (il y a normalement fracture de ce qui serait la partie bulbaire dans un éclat bipolaire) ; quant aux extrémités distale et 


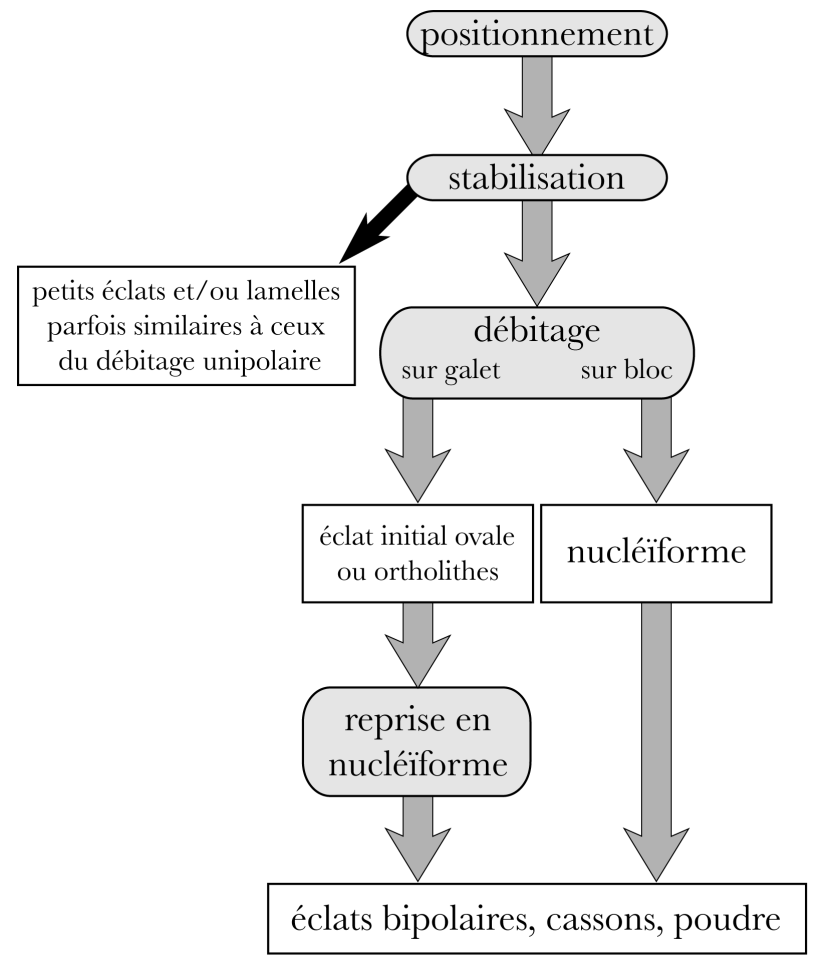

Figure 8 - Représentation schématique des étapes $d u$ débitage « bipolaire » sur enclume.

Figure 8 - Schematic representation of the stages of «bipolar » on anvil flaking. proximale, ce sont des «talons " linéaires et/ou punctiformes écrasés. Dans le cas du quartz, la partie de la pièce nucléiforme frappée par le percuteur est souvent punctiforme (partie proximale conique), alors que la base posée sur l'enclume est plus fréquemment linéaire (droite ou virgulée). De nombreux produits sont biconiques avec deux talons punctiformes (surtout les pièces nucléiformes en fin de débitage) et d'autres sont plus ou moins rectangulaires (particulièrement dans le cas des roches plus résistantes comme le silex), étroits et présentent deux longs « talons" linéaires opposés : ce sont les objets que la bibliographie appelle « pièces esquillées ».

En conséquence, le percuteur garde généralement des marques profondes, surtout punctiformes, alors que les stigmates laissés sur l'enclume sont plus souvent virgulés et plus ras.

Certains produits de débitage traversent tout le nucléiforme, car le débitage sur enclume permet d'obtenir facilement des éclats aussi longs que le bloc à débiter ; ils présentent donc un double talon écrasé. Les produits qui n'ont pas traversé - particulièrement ceux qui ont été détachés par des coups légèrement obliques par rapport à l'axe de la pièce à débiter - peuvent présenter un talon (unique) linéaire ou punctiforme sans marque d'écrasement très visible et être parfois confondus avec des éclats de débitage unipolaire. C'est aussi le cas des esquilles et des éclats qui sortent latéralement au moment de la stabilisation. II faut donc être prudent quand on a affaire à des industries utilisant la technique bipolaire, réservant une catégorie spéciale pour les pièces dont l'origine de débitage est « douteuse ».

Si cette technique est appliquée au quartz, les déchets peuvent être très nombreux, surtout quand on a peu d'expérience (jusqu'à $30 \%$ de poudre et micro débris polyédriques, lors de nos premiers essais) ou quand la matière est très saccharoïde. On apprend vite à diminuer le gâchis en « sentant » (par les doigts) et « écoutant » (par l'oreille) le bloc, en le tenant bien ferme, en le comprimant avec les doigts pour diriger les ondes de choc et en dosant la force de percussion. Le silex produit moins de poudre et plus de nucléiformes rectangulaires que le quartz; cette matière, pour sa part, laisse surtout des pièces nucléiformes biconiques fusiformes.

On arrive finalement à être assez conscient des produits qui vont sortir ; il est possible d'obtenir assez facilement des produits laminaires ou lamellaires (surtout en utilisant les angles qui délimitent les facettes des cristaux de quartz) ce qui peut suggérer à l'archéologue l'existence de débitage lamellaire classique dans des industries de quartz; nous l'avons remarqué aussi bien en Galice que dans le Brésil central (fig. 10) ; l'examen des photographies des lamelles de quartz de Zitny (Moravie) publiées par Jelinek (1976) nous suggère qu'il s'agit bien de produits « bipolaires ». Nous verrons que l'on peut obtenir aussi des éclats initiaux de forme déterminée sur des galets ovoïdes.

De toute façon, le contrôle est en général très relatif et la technique bipolaire revient surtout à produire un grand 


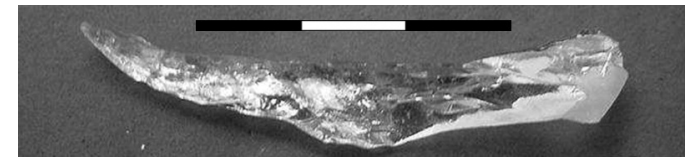

Figure 10 - Lamelle de quartz extraite expérimentalement sur enclume.

Figure 10 - Bipolar experimental blade.
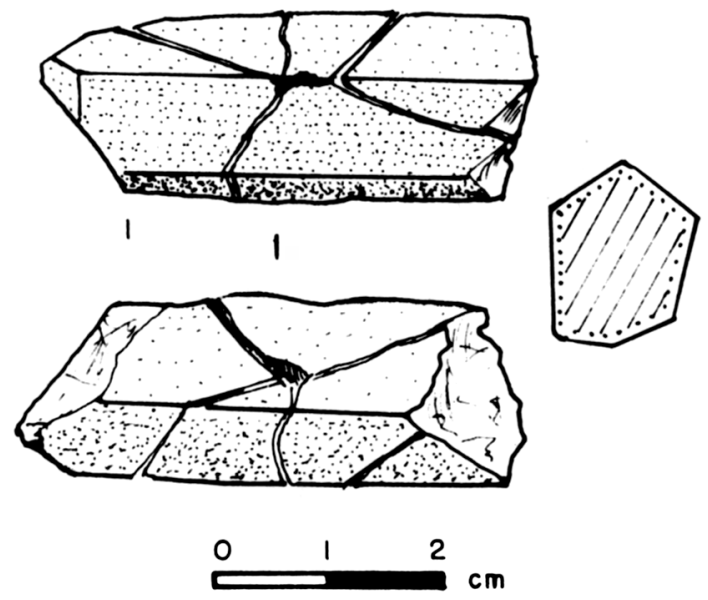

Figure 11 - Fracture en étoile sur un cristal de quartz.

Figure 11 - Star fracture on crystal. nombre de fragments, parmi lesquels on trie ceux qui présentent les caractéristiques désirées (éclats et/ou nucléiformes). En effet, les amas de débitage bipolaire offrent toute la gamme désirable de tranchants bruts plus ou moins aigus ou robustes, de dos naturel, voire d'extrémités pointues directement utilisables comme perçoirs.

Dans le centre de Minas Gerais, des cristaux ont été aussi fracturés couchés sur l'enclume ; cela provoque une fracture en étoile (fig. 11) (ou « en aile de papillon » selon l'expression de $\mathrm{J}$. Pelegrin) qui génère quatre fragments de forme typique.

Pour différencier, sur les dessins, les pièces débitées sur enclume, nous avons adopté un symbole spécial, placé au-dessus du talon écrasé des éclats ou de la ligne d'attaque des pièces nucléiformes, et qui évoque la multiplicité des coups reçus (fig. 12 b).

\section{Les positions et les gestes}

Selon que l'on veut fendre une pièce épaisse (galet, bloc ou ancien nucléus), débiter des lames latérales ou des éclats longitudinaux, façonner une pièce bifaciale ou retoucher un tranchant, la position relative de l'enclume et de la masse travaillée varie, ainsi que le geste du tailleur et l'angle de percussion :

- pour fendre (recherche d'une fracture « en split ») : la masse à débiter est placée (verticalement ou horizontalement) sur une surface plane de l'enclume, la percussion - violente est portée verticalement avec une surface plane ou peu convexe du percuteur ;
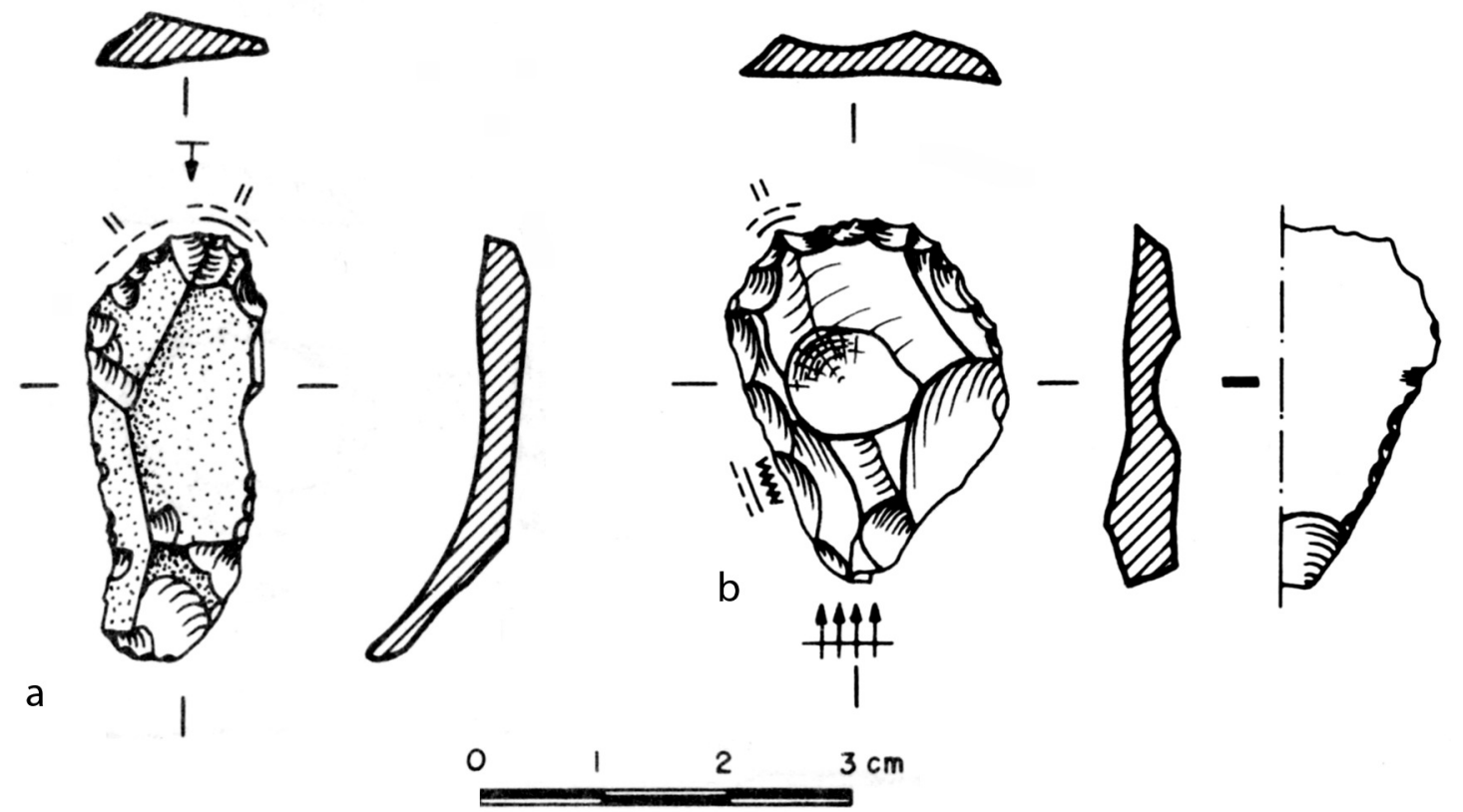

Figure 12 - Grattoirs de cristal de roche sur éclats unipolaire (a) et bipolaire (b). Les deux proviennent de la même couche dans l'abri de Santana do Riacho.

Figure 12 - End scrapers on unipolar (a) and bipolar (b) flakes, from the same layer of Santana do Riacho shelter. 

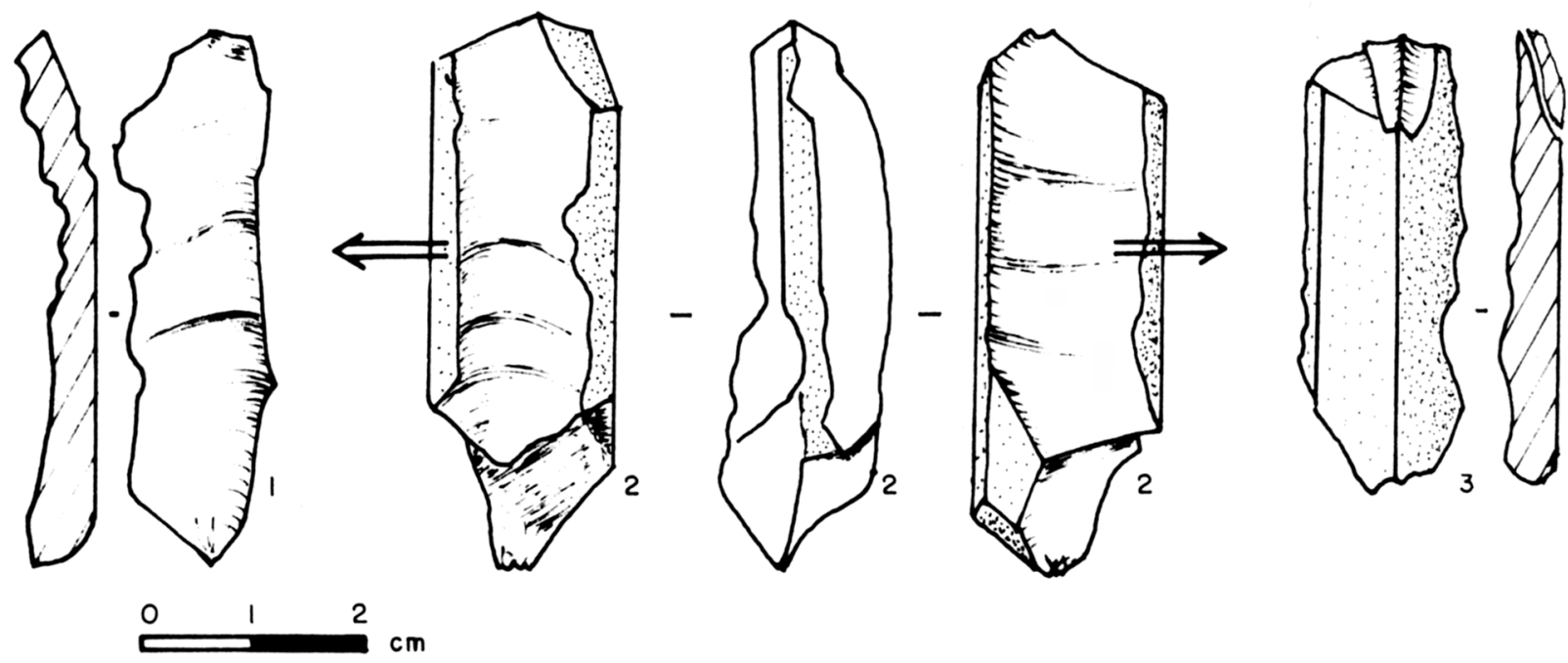

Figure 13 - Production sur enclume de lames épaisses à partir d'un cristal de quartz.

Figure 13 - Thick blades bipolar production on quartz crystal.

- pour obtenir un éclat initial ovale à partir d'un galet, on l'inclinera très légèrement et la percussion doit être plus modérée ;

- pour tenter d'obtenir un produit laminaire latéral à partir d'une pièce nucléiforme rectangulaire, la position relative de l'enclume et du support est la même mais le coup est porté obliquement, pour frapper un dièdre latéral et non l'ensemble de la ligne écrasée du talon ;

- pour favoriser l'obtention de produits laminaires longitudinaux minces le long d'un monocristal, il faut avoir retiré la couronne, puis placer le prisme légèrement en oblique et frapper au sommet d'une des arêtes naturelles formées par la rencontre des facettes du cristal. Si le prisme est placé verticalement, on peut obtenir un produit beaucoup plus épais (fig. 13).

\section{Le façonnage et la retouche}

La technique de taille sur enclume n'est pas réservée au débitage : elle s'applique aussi au façonnage. Nous avons identifié et reproduit la fabrication de préformes de lames de hache par taille bifaciale sur enclume, à partir de blocs, gros éclats et de galets de grès silicifiés dans les sites de la région de Manaus (Costa 2003) et le nord du Maranhão (collections réunies par l'équipe de S. Caldarelli) ; l'objet est alors appuyé sur l'enclume, mais légèrement incliné, (fig. 14) le coup aussi est porté obliquement, pour éviter de fendre la pièce. Les éclats qui sortent ont alors un tranchant linéaire écrasé (typiquement bipolaire) mais la face interne ressemble à celle d'un éclat unipolaire.

II s'agit alors d'une technique intermédiaire entre la taille à main libre et la taille sur enclume, que l'on pourrait appeler « taille appuyée sur enclume ».

Nous n'insisterons pas sur la retouche dite " écrasée ", très efficace pour la création de certains bords abattus. Elle est souvent obtenue par percussion sur une enclume ; la partie à abattre est alors appuyée sur un bord dièdre de l'enclume et non pas sur une face d'appui. La même position est utilisée quand on veut fractionner une lame pour produire des microlithes.

Le percuteur est alors beaucoup plus petit que dans les cas précédents et le geste limité à un mouvement du poignet ; en effet, ce qui importe n'est plus la puissance du choc mais la précision de l'impact. Les risques de cassures sont importants ; J. Pelegrin (communication personnelle) les réduit en utilisant une enclume en bois.

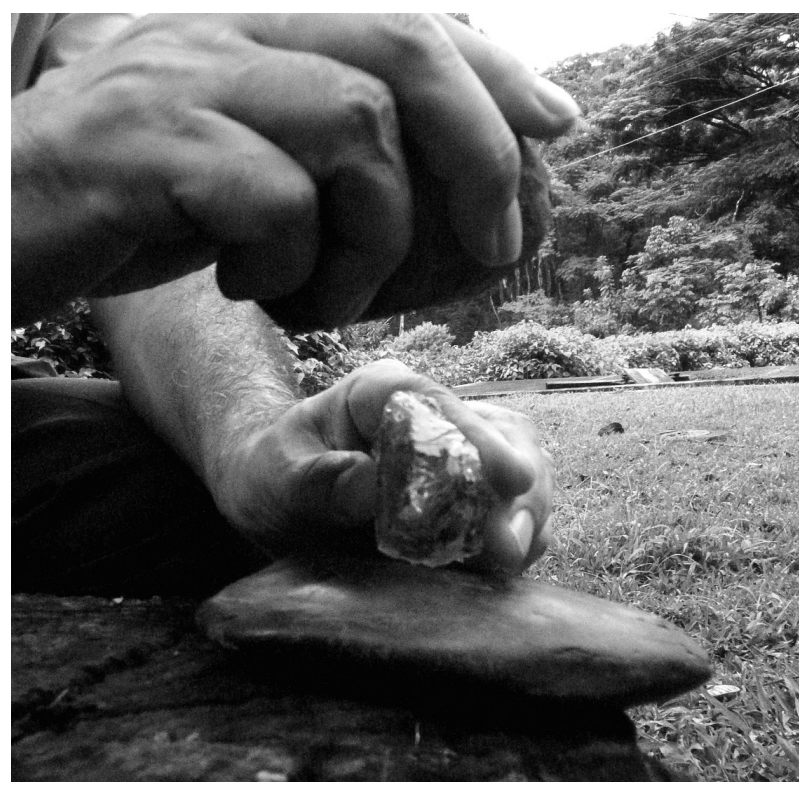

Figure 14 - Ébauche de lame de hache préparée avec appui sur enclume.

Figure 14 - Blade for an axe, being shaped on anvilstone. 

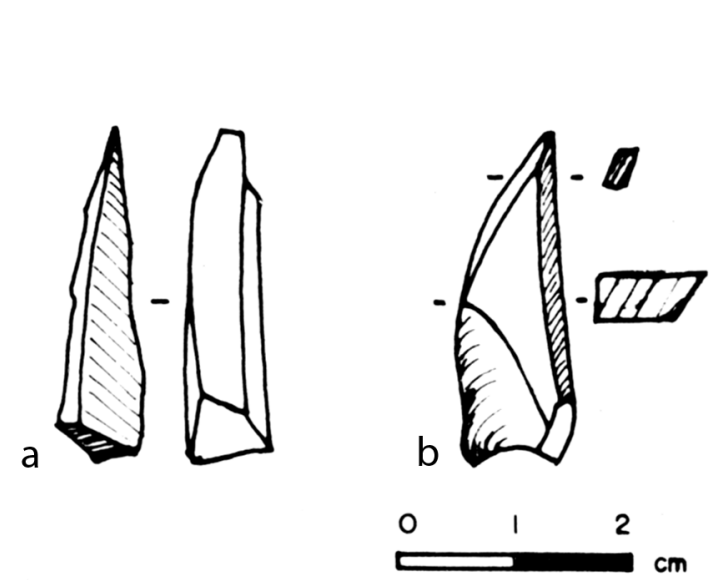

D'autres types de travail sur enclume sont théoriquement possibles et nous avons vu J. Tixier s'amuser à retirer des chutes de burin d'angle sur enclume, percutant la pièce au milieu du tranchant, le contrecoup sur l'enclume faisant filer l'éclat... Mais d'une manière générale, la retouche à main libre est plus simple et permet de mieux contrôler le résultat, encore que la retouche de bord appuyée sur enclume puisse être utile quand on travaille des roches relativement tenaces comme le basalte.

Les produits du débitage bipolaire sont rarement façonnés ou retouchés car, comme nous l'avons dit plus haut, cette technique de débitage produit facilement tous les types de tranchants désirables : très aigus (les éclats minces), semiaigus ou abrupts (particulièrement les pièces nucléiformes), abrupts (la fréquence des fractures de type Siret est grande, particulièrement quand on travaille le quartz), permettant de couper, racler, gratter ou de disposer de dos naturels. Les esquilles en formes d'aiguille fournissent d'excellents perçoirs, alors que les bâtonnets ont des fils et des coins semblables aux burins (fig. 15).

II apparait donc que les producteurs des industries « traditionnelles » d'Europe, du Moyen-Orient et d'une bonne partie de l'Afrique qui travaillent en " mode unipolaire » investissent dans la retouche pour une raison d'esthétique traditionnelle et non parce que cela permettrait une plus grande efficacité technique ou fonctionnelle.

\section{Quelques erreurs de diagnostic}

Nous n'avons jamais produit d'éclats avec un bulbe à chaque extrémité de la même face, au contraire de ce que mentionne Kobayashi (1975). Pour obtenir un tel éclat, il faut percuter un plan de frappe sans détacher l'éclat, retourner le nucléus et frapper à l'opposé ; avec beaucoup de chance, il sortira un éclat à deux bulbes symétriques (cela nous est arrivé une seule fois, accidentellement), obtenu par taille à main libre.

Quant à l'éclat à « bulbe interne mésial » observé et décrit par T. Miller (1975), produit lors du débitage d'un bloc sur enclume par un Indien Xeta du Paraná (Brésil) et qu'il a cru caractéristique du débitage bipolaire, nous avons pu vérifier qu'il
Figure 15 - Aiguilles bipolaires utilisables comme perçoirs $(a, b)$ et baguette utilisable comme burin (c).

Figure 15 - Bipolar products that may be used as drills $(a, b)$ or burin (c). s'agissait d'un éclat thermique « virtuel » en formation, effectivement détaché lors du travail sur enclume : le centre de l'éclat est la partie la plus épaisse et présente un petit renflement qui a été confondu avec un bulbe par ce chercheur.

Certains auteurs peu habitués aux industries de quartz ont cru reconnaitre des grattoirs retouchés alors qu'ils observaient des éclats à talon écrasé ou des pièces nucléiformes à extrémité écrasée (Hurt 1960 ; Hurt et Blasi 1969, dans les industries brésiliennes de Lagoa Santa). Les petits éclats courts qui sortent des parties percutées («talons » bipolaires caractéristiques) peuvent en effet être confondus avec des fronts retouchés par un observateur non averti.

De notre côté, dans un article préliminaire sur l'archéologie de Santana do Riacho (Prous 1981) nous avions interprété certaines pièces nucléiformes (fig. 16) comme de vrais nucléus à lamelles, avant de vérifier que le retrait non contrôlé d'enlèvements lamellaires était un phénomène spontané assez fréquent.

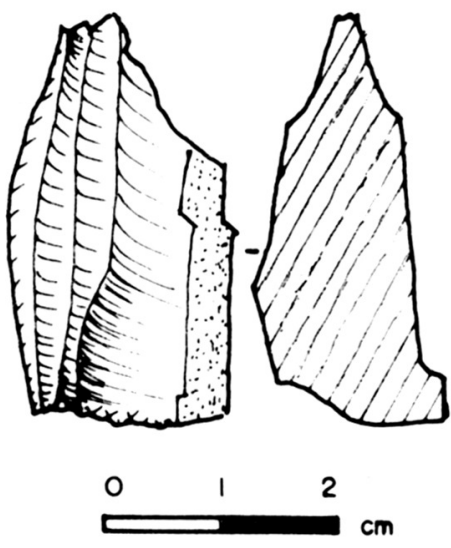

Figure 16 - Pseudo nucléus à lamelle de Santana do Riacho : en fait il s'agit d'une pièce nucléiforme typique.

Figure 16 - This typical nucléiform product (bipolar core) from Santana do Riacho looks like a core for bladelets. 
Des éclats et des lames d'apparence « unipolaire » peuvent provenir de coups portés latéralement sur les pièces nucléiformes. Nous avons obtenu expérimentalement de telles lames courbes sur quartz au Brésil (fig. 10), et une étudiante en a produit une très belle en silex lors du cours que nous avons donné en Galice en 2003. On peut normalement les reconnaître, soit que leur face supérieure présente des stigmates perpendiculaires à l'axe de débitage les faisant ressembler à des lames à crête (si le support a été retourné de $90^{\circ}$ ), soit que leur talon porte des stigmates d'écrasement.

J. Flenniken (1981) a cru pendant un temps que le débitage bipolaire provoquait des ondes extrêmement prononcées ; les expériences que nous avons faites avec lui en 1984 sur du quartz (hyalin ou non) et sur certaines silexites ont montré qu'il n'en est rien. Bien que quelques pièces de quartz montrent effectivement de tels stigmates, il s'agit d'exceptions, surtout rencontrées sur les petits éclats de stabilisation (ou à l'emplacement de leurs négatifs, sur les pièces nucléiformes), bien que nous l'ayons parfois observé lors du débitage de cristaux (fig. 13) ; en fait, c'est l'absence d'ondes, sur une face souvent très plane, qui caractérise le mieux les grands éclats extraits sur enclume.

Finalement, on ne doit pas oublier que des pièces esquillées peuvent se former spontanément par concassage dans les endroits riches en débris de quartz (action des machines travaillant dans les gravières, par exemple).

\section{3 - VARIABILITÉ DES PRODUITS DE TAILLE BIPOLAI- RE DANS DIFFÉRENTES RÉGIONS}

\section{Minas Gerais (Brésil central)}

\section{Lagoa Santa/ Serra do Cipó/Serra do Espinhaço}

Bien qu'il existe dans cette région des cristaux de très grandes dimensions (jusqu'à près d'un mètre de hauteur !), les outils de quartz étaient surtout produits à partir de cristaux de taille modeste. Au début de l'Holocène, les éclats provenant de la couronne des cristaux étaient souvent détachés par percussion à main libre et servaient de support à des grattoirs très soignés de deux à trois centimètres de longueur (fig. 12). Le débitage continuait ensuite sur enclume, permettant éventuellement d'obtenir des enlèvements laminaires le long des arêtes et surtout, des éclats. Dans le cas de très grands cristaux très hyalins, la taille sur enclume et celle à main libre alternent parfois : unipolaire à partir de plans cristallins, bipolaire quand on ne disposait pas de surface cristalline inclinée. D'une manière générale cependant, la taille bipolaire semble avoir été responsable de la plupart des éclats et les nucléus sont très rares par rapport aux pièces nucléiformes.

\section{Vallée du Rio Doce}

Dans les sites tupiguarani du Rio Doce, la taille fut pratiquée presque exclusivement sur des blocs de quartz polycristallins de taille décimétrique et non sur des monocris-

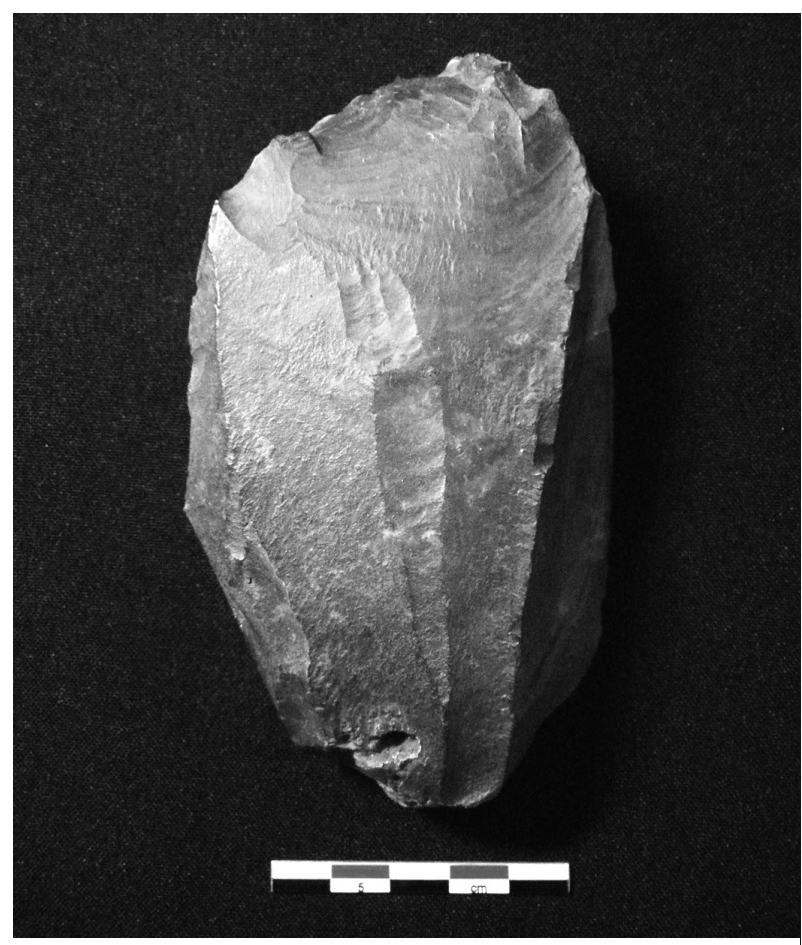

Figure 17 - Pièce de jaspe débitée sur enclume, site Vargem Grande (vallée du rio Peruaçu).

Figure 17 - Bipolar jasper core from Peruaçu Valley (site Vargem Grande).

taux. Ce quartz polycristallin présente de nombreuses fractures parallèles entre elles, qui résultent des pressions reçues par le quartz pris entre des blocs de roche magmatique encaissante progressant selon des mouvements de translation. En conséquence, les tentatives d'extraction d'éclats par percussion libre provoquent souvent des cassures mésiales au niveau des plans de fracture, que la percussion bipolaire permet d'éviter car les plans de partition traversent alors les cristaux et franchissent les discontinuités virtuelles.

Les éclats bipolaires du Rio Doce présentent donc des dimensions moyennes bien supérieures à celles de Santana, et nombreux sont ceux qui atteignent entre cinq et sept centimètres de longueur (fig. 17). Aucune de ces pièces ne montre la moindre retouche. Notons que les éclats à apparence unipolaire ou d'identification douteuse sont assez nombreux dans ces sites, alors qu'on ne trouve pratiquement aucun nucléus, mais de nombreuses pièces nucléiformes - certaines encore de grandes dimensions (12 cm dans le site Florestal 2). II semble donc que la part de la technologie bipolaire puisse être sous-estimée dans certaines collections au simple vu des éclats.

\section{Vallée du Peruaçu et du Moyen São Francisco}

Nous avons remarqué la présence de quelques ensembles de silex travaillés par débitage bipolaire dans un abri et 


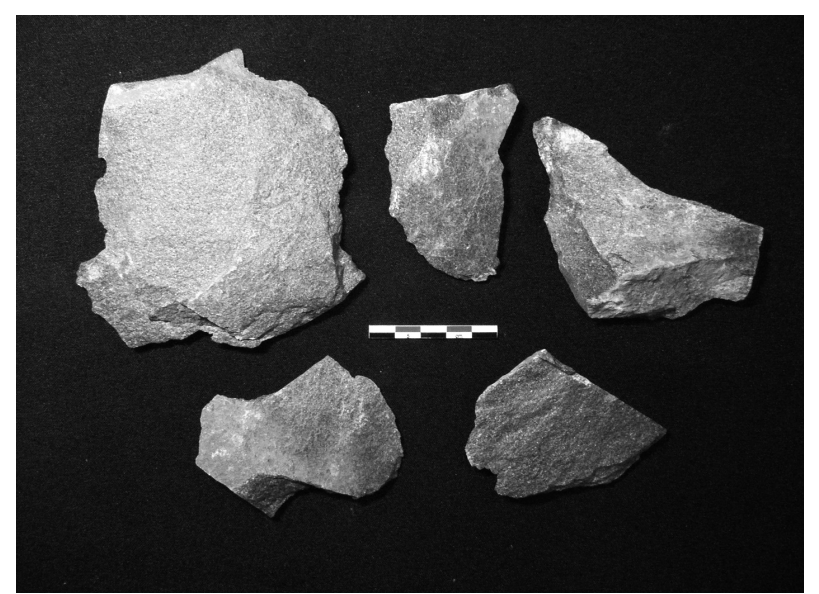

Figure 18 - Éclats expérimentaux très plats et fins retirés pour amincir sur enclume une préforme de lame de hache en roche basique reproduisant les produits préhistoriques de Monte Alegre.

Figure 18 - Experimental bipolar thinning flakes produced on anvilstone ; reproduction of archaeological products from Monte Alegre (greenstone).

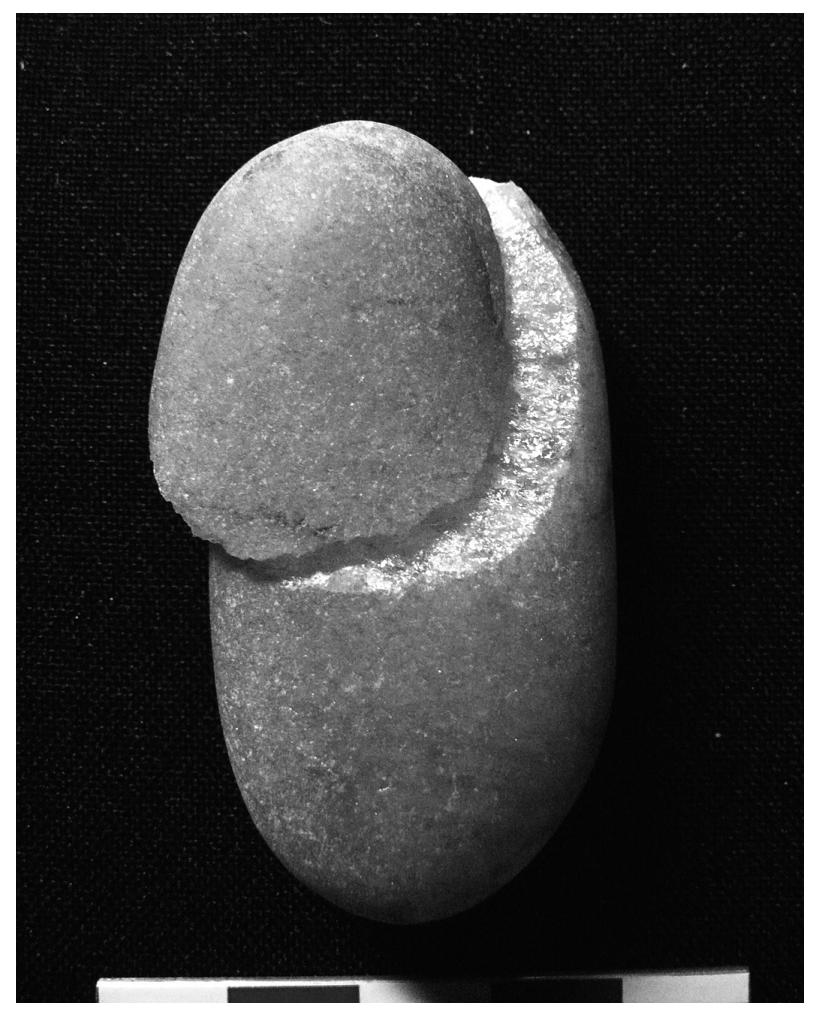

Figure 19 - Éclat initial ovale sur galet (expérimental).

Figure 19 - Oval initial flake from a pebble (experimental). dans le site tupiguarani de Vargem Grande (fig. 17) mais cette technique a été particulièrement appliquée dans le seul gisement où la présence du quartz est importante (Russinhos).

\section{Monte Alegre de Minas}

En étudiant le matériel recueilli dans l'atelier de fabrication de lames de haches polies en roche basique de Monte Alegre (ouest de Minas Gerais), nous avons trouvé des éclats d'amincissement très plats que nous avons reproduit avec plusieurs techniques ; en particulier, nous avons montré que la ténacité de certaines roches vertes - tels les gabbros - permet de les façonner sur enclume sans que le talon (souvent punctiforme) montre de traces d'écrasement (fig. 18).

\section{Le débitage sur enclume des galets dans le Brésil central}

On trouve des débitages classiques de galets le long du rio São Francisco : tranches de saucisson et quartiers d'orange en quartzite à Buritizeiro (Minas Gerais) et de jaspe dans les dunes d'Itaparica (état de Bahia). Mais des formes particulières ont été recherchées dans certaines industries de l'état de Goias, où les préhistoriques du début de l'Holocène ont cherché à obtenir sur enclume de grands éclats initiaux ovales retirés de galets de quartzite à faible courbure (fig. 19). Certains d'entre eux ont été produits sur enclume en évitant de frapper trop fort et verticalement - ce qui fendrait le galet, provoquant la formation d'un hémilite (selon la nomenclature de Van Riet Lowe, cité par Brézillon 1977; cf. fig. 3). L'éclat, entièrement cortical, subit ensuite un façonnage inverse. L'objectif était d'obtenir un outil plan-convexe uniface, dont la face interne seule était retouchée, alors que la partie corticale lisse, laissée intacte, servait de face plane - comme le serait la face interne d'un éclat épais transformé en rabot. On retrouve dans les mêmes séries quelques seconds éclats (en " tranche de pain ") obtenus de la même manière, immédiatement après le retrait de l'éclat initial.

\section{Les industries d'agate du sud brésilien (États de Santa Catarina et Rio Grande do Sul)}

Nous avons observé que si le débitage du quartz sur enclume produit un grand nombre de pièces nucléiformes biconiques, celui du silex crée surtout des formes rectangulaires aplaties (les typiques "pièces esquillées "). Alors que nous étudiions le traitement sur enclume des agates par les groupes tupiguarani de Santa Catarina, le géologue Joël Quémeneur nous a montré que les tailleurs percutaient les pièces dans le sens des fibres de calcédoine pour obtenir des produits allongés - en majorité, des aiguilles (fig. 20), mais aussi des pièces nucléiformes rectangulaires extrêmement plates et étroites (fig. 21) qui correspondent en fait à des tronçons de section quadrangulaire provenant du fractionnement longitudinal spontané des éclats selon un processus de type Siret multiple, selon des plans parallèles. 


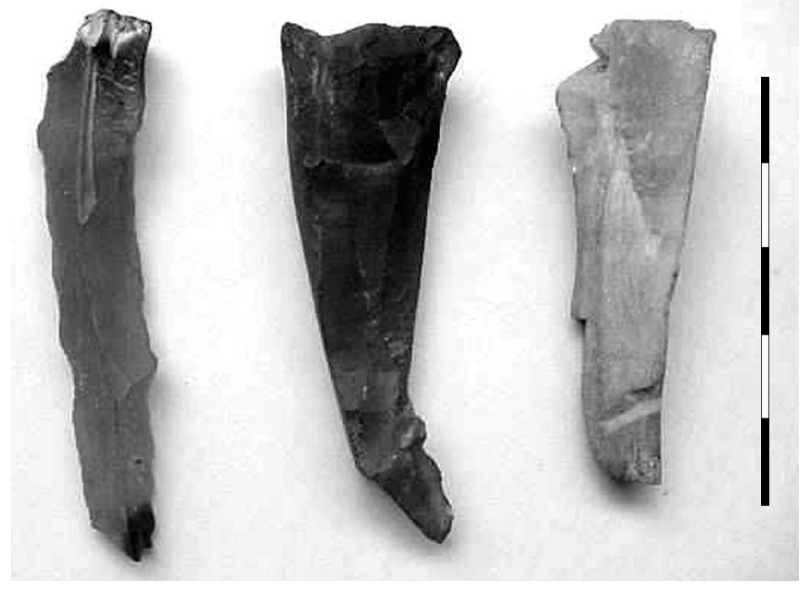

Figure 20 - Aiguilles typiques du débitage de l'agate dans l'état de Santa Catarina.

Figure 20 - Typical needles of agata industries from Santa Catarina state.

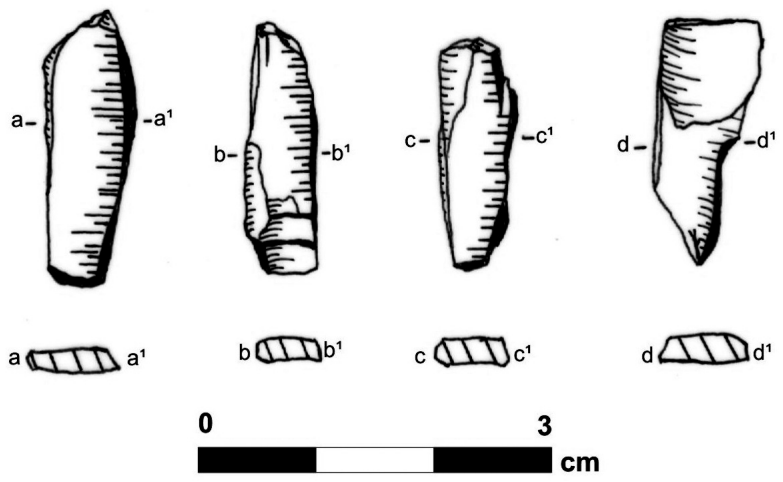

Figure 21 - Pièces rectangulaires fines typiques du débitage de l'agate dans l'état de Santa Catarina.

Figure 21 - Typical rectangular thin products of agata industries from Santa Catarina state.

\section{Amazonie (taille appuyée sur enclume des lames de} hache/ râpes à manioc)

\section{Façonnage des préformes de hache}

L'industrie de pierre amazonienne est encore presque totalement inconnue mais nous avons pu avoir accès aux premières collections recueillies au cours de ces trois dernières années par l'équipe de Scientia dans le Maranhão et par celle de l'Université de São Paulo près de Manaus.

Nous avons alors vérifié que, dans ces deux régions, des préformes de hache en quartzite avaient été façonnées sur enclume (fig. 22, 23). II s'agit de bifaces épais, que nous avons pu reproduire expérimentalement à partir de blocs

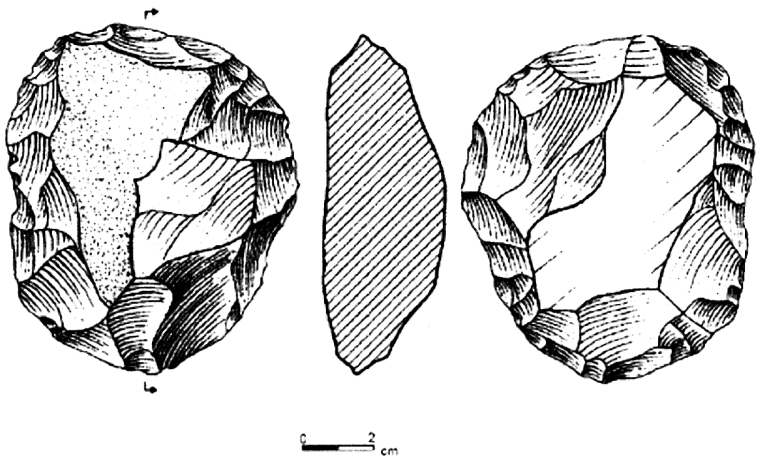

Figure 22 - Préforme pour lame de hache en quartzite, façonnée sur enclume (Manaus, Amazonie). Le dessinateur n'a pas représenté l'écrasement des bords ; d'après Costa 2003.

Figure 22 - Prehistoric preform for ground axe, shaped on anvilstone (Manaus, Amazonia) ; silicified sandstone. The drawing doesn't show the crushing of the edges ; from Costa 2003.

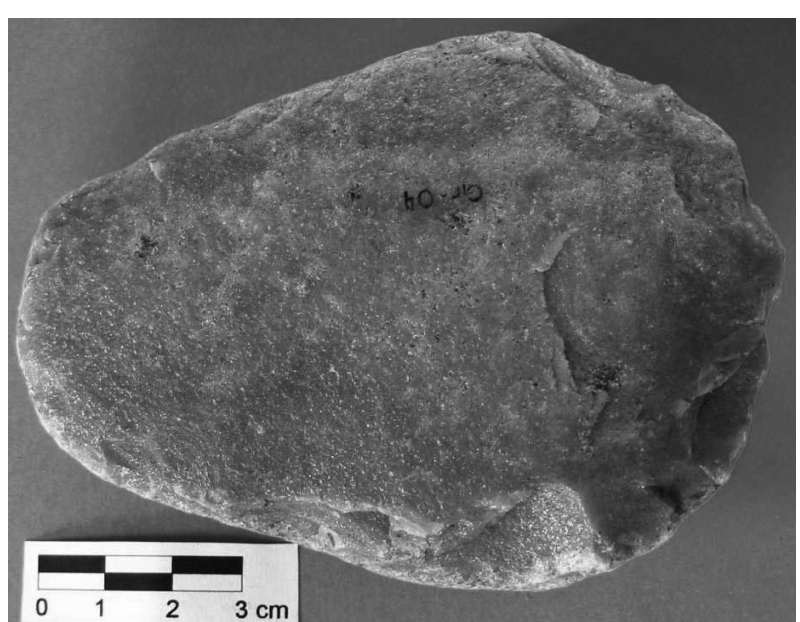

Figure 23 - Préforme pour lame de hache, état du Maranhão (document de l'entreprise de sauvetage archéologique Scientia).

Figure 23 - Preform for a ground axe, Maranhão state (photograph : Scientia).

de quartzites ramassés près de Manaus. Pour éviter que les pièces ne se fendent sur toute leur largeur, nous avons incliné les préformes, réalisant une percussion " oblique appuyée sur enclume » et non la percussion verticale, propre au débitage bipolaire. Les arêtes présentent un écrasement typique qui ressemble aux stigmates d'un bouchardage, et les éclats de façonnage restent suffisamment marginaux pour ne pas diminuer l'épaisseur et la résistance de l'objet. Nous avons ensuite vérifié que les époux Pétrequin avaient également observé une technique de façonnage sur enclume semblable dans l'Irian Jaya (Pétrequin et Pétrequin 1993). 


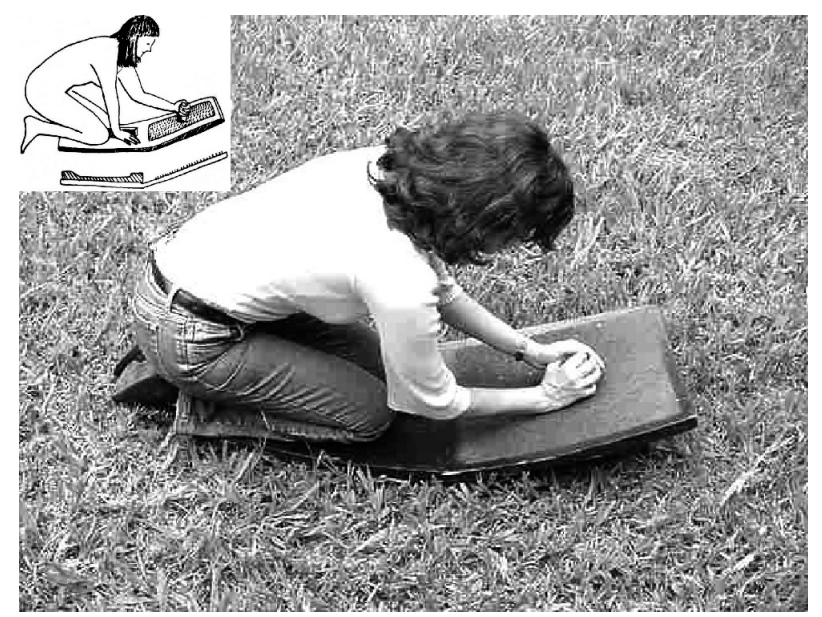

Figure 24 - Râpe à manioc Baniwá ; Rio Negro.

Figure 24 - Baniwa Indians manioc rasp ; Rio Negro river.

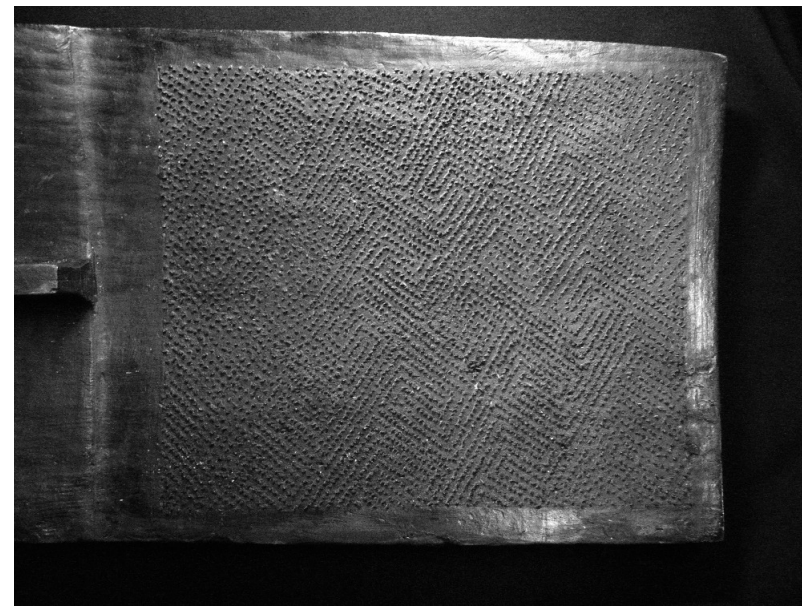

Figure 25 - Les dents de pierre de la râpe forment un dessin en quinconce qui permet d'augmenter leur efficacité.

Figure 25 - The disposal of the teeth improve the efficiency of the manioc rasp.

\section{Production des dents de racloir à manioc Baniwa}

Les indiens Baniwa de haute Amazonie fabriquent (et vendent aux communautés qui vivent tout au long des affluents du Rio Negro) des râpes à manioc formées par une planche de bois tendre (fig.24, 25) dans laquelle sont plantés des petits éléments aigus de quartzite ou de gneiss (selon l'ethnologue B. Ribeiro), de diabase selon l'identification pétrographique faite sur notre exemplaire ethnographique par le géologue J. Quémeneur. D’après Reichel-Dolmatoff (1997), les Desana de Colombie utiliseraient des dents en quartz pour ce même usage. Nous avons parlé - avec difficulté, car ils ne connaissent guère le portugais - avec des hommes Baniwa pour savoir comment étaient fabriquées ces dents, mais ils répondirent

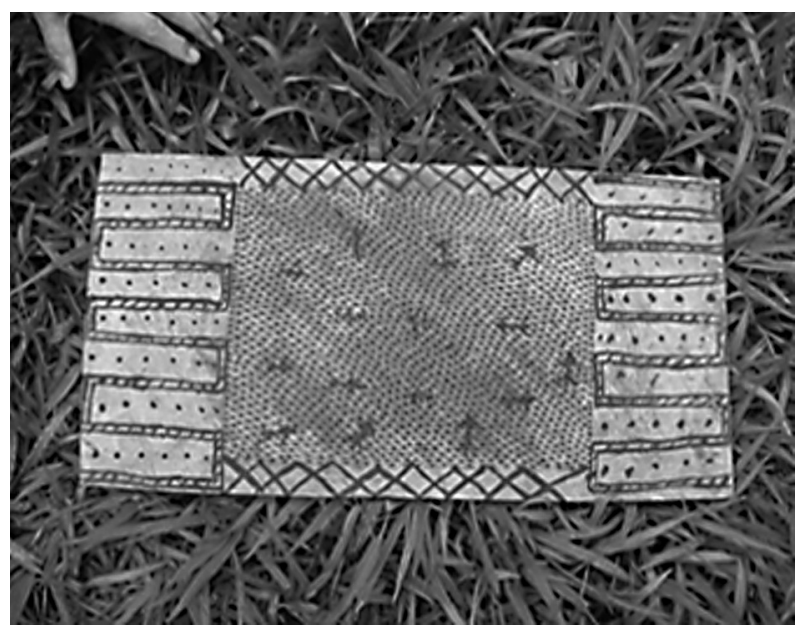

Figure 26 - Râpe à manioc Wai Wai (Guyane brésilienne).

Figure 26 - Wai Wai Indians manioc rasp (brazilian Guyana).
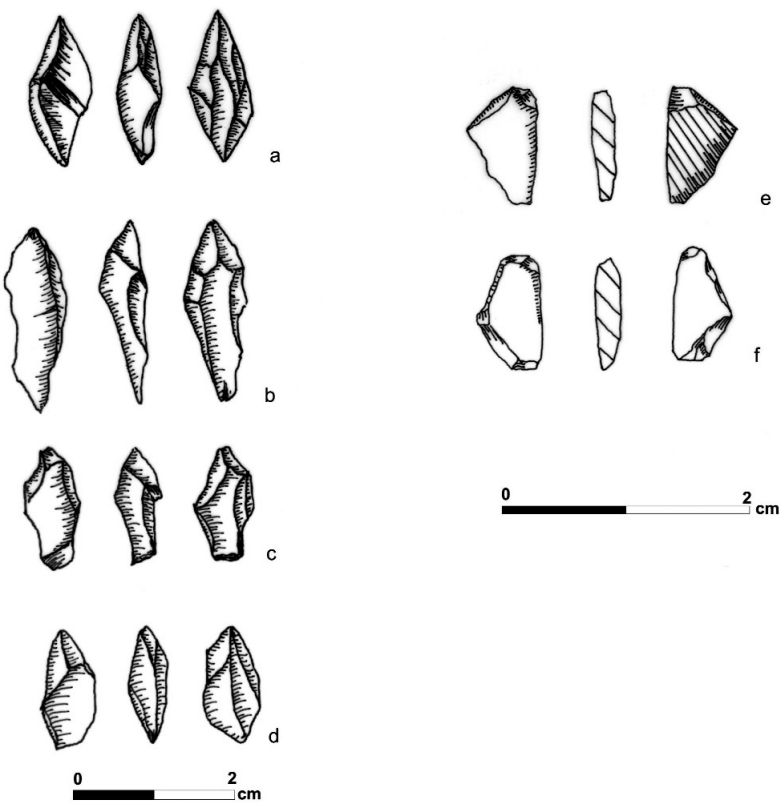

Figure 27 - Dents de râpe à manioc expérimentales $(a, b$ : produits de concassage du quartz avant retouche; $c$ : après retouche par pression) ; $d$ : dent en diabase provenant d'une râpe Baniwá).

Figure 27 - Experimental quartz teeth for manioc rasp (a, $b$ : bipolar products before retouching ; $c$ : after retouching); $d$ : ethnographic tooth (diabase) from Baniwa rasp. 
tous que c'était un travail féminin et qu'ils ne savaient rien à ce sujet. Les Wai Wai de la Guyane brésilienne fabriquent également des râpes à dents de pierre (fig. 26). La morphologie et la faible dimension de ces pointes nous ont suggéré qu'elles ne pourraient être obtenues que par percussion bipolaire. Nous avons effectivement pu reproduire facilement ces mêmes formes par une percussion sur enclume de pierre (fig. 27). Les expériences faites par l'un de nous (F. A.) sur quartz, silex, agate, diabase, quartzite et gneiss ont montré que toutes ces matières fournissaient par concassage sur enclume des fragments utilisables, éventuellement après un égrisage effectué par raclage sur enclume, surtout nécessaire pour le quartz, destiné à renforcer la pointe active. Ils sont ensuite disposés en quinconce et insérés dans des encoches (ouvertes avec un ciseau en os lors de nos expériences).

\section{4 - « LA PIÈCE ESQUILLÉE : OUTIL OU DÉCHET? » - EXPÉRIENCES D'UTILISATION ET TENTATIVES D'OBTENTION PAR UTILISATION COMME COIN}

J. Flenniken pensait que les pièces esquillées pourraient se former par utilisation d'un éclat robuste comme coin pour fendre du bois. A. Ravere (in Swanson 1975) considérait déjà qu'elles étaient excellentes pour cet usage, alors que Dickson (1977), un ingénieur très objectif, ne leur trouve aucun avantage ; Mazière (1984) posait clairement la question : la pièce esquillée était-elle un outil ou un déchet?

Nous avons, quant à nous, vérifié que des éclats robustes (aussi bien obtenus à main libre que sur enclume) ou des pièces nucléiformes étaient fort efficaces pour fendre des branches d'une dizaine de centimètres de diamètre.

Les expériences (réalisées par F. A. et G. S.) montrent que les pièces esquillées sont efficaces comme ciseaux pour creuser des cavités dans du bois assez tendre (préparation d'un manche pour une lame polie) mais seulement jusqu'à une faible profondeur, puisqu'elles ne sont jamais très allongées ; elles sont ainsi insuffisantes pour terminer la perforation d'un manche de type direct mâle destiné à recevoir une lame triangulaire (système le plus courant dans le Brésil central, cf. Prous et al. 2002), mais permettent parfaitement de creuser la dépression nécessaire pour une hache à tenon semblable à celle que les Masheo de l'Amazonie péruvienne utilisaient encore vers 1970 (A. Distel 1972-1973).

Cette même restriction vaut pour l'usage comme coin. Dans ce cas, l'extrémité frappée avec un percuteur en pierre présente à la fois des marques de piquetage et des éclatements semblables à celui que provoque la taille sur enclume (fig. 28). Son apparence est donc fort distincte de celle des pièces esquillées classiques. L'extrémité active opposée, par contre, est assez semblable à celles de pièces esquillées communes et présente souvent des enlèvements fortement rebroussés (fig. 29).

Des éclats robustes de quartz, de silex et d'agate obtenus par percussion libre (unipolaire) se sont montrés égale- ment très efficaces pour fendre des branches d'une dizaine de centimètres de diamètre. Les formes les plus efficaces ont été des éclats larges, à talon épais et tranchant assez aigu ; quand cette forme n'était pas celle de la pièce originale, elle se formait progressivement au cours du travail. Les tranchants bruts se sont parfois cassés mais ne se sont que rarement machurés. Par contre, l'extrémité percutée à la pierre s'esquillait fortement, formant parfois une concavité prononcée rappelant une coche robuste (fig. 30) - une caractéristique que nous avons observée également dans l'industrie de Santa Catarina et aussi sur quelques pierres à fusil. L'enfoncement des coins par un percuteur en bois, par contre, n'a laissé que fort peu de stigmates.

On voit donc que, d'une manière générale, les pièces d'origine unipolaire utilisées comme coin, malgré les stigmates qu'elles portent, ne se confondent pas avec des nucléiformes bipolaires ou avec des " pièces esquillées " classiques.

Rappelons enfin, que les pierres à fusil - taillées aussi bien en quartz qu'en silex - fabriquées dans des ateliers familiaux au Brésil jusque vers les années 1930, sont des éclats quadrangulaires qui sont percutés et retournés en cours d'usage. Cela leur laisse une morphologie et des stigmates d'écrasement sur deux lignes opposées (fig. 31) qui les rapprochent fort des "pièces esquillées " classiques.

\section{5 - L'ENCLUME ET LE MARTEAU}

\section{Le marteau...}

Nous avons vu que, dans tous les cas observés au Brésil, le percuteur pour débitage bipolaire porte ses stigmates sur une face et non aux extrémités (comme c'est le cas pour les percuteurs unipolaires) ou en périphérie (cas fréquent dans les bouchardes brésiliennes) (fig. 32). Ces stigmates peuvent se trouver presque au centre d'une ou de deux faces opposées d'un percuteur massif (c'est le cas dans le Minas Gerais où les percuteurs sont des galets ovoïdes) ou fortement décentrés sur un percuteur allongé (Rio Grande do Sul, cf. Schmitz et al. 1990, où il s'agit de galets provenant probablement de colonnes de basalte).
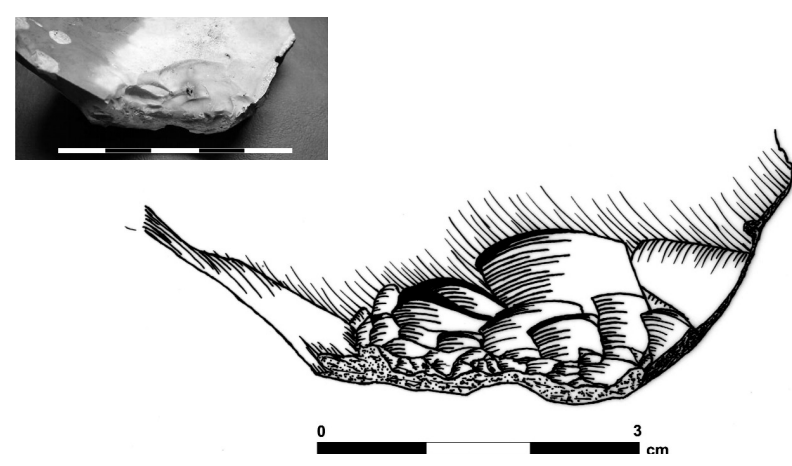

Figure 28 - Éclat utilisé comme coin ; vue du talon percuté.

Figure 28 - Flaked used as a wedge : the stroken butt. 


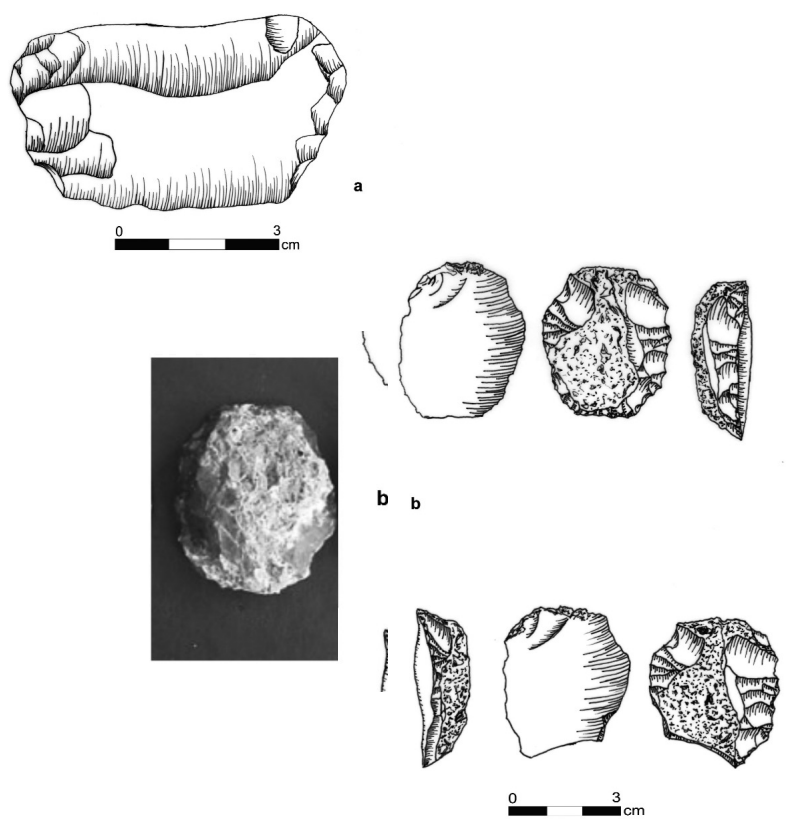

Figure 29 - Éclat de quartz utilisé comme coin, montrant des marques d'utilisation semblables à celles de pièces esquillées.

Figure 29 - Quartz flake used as a wedge ; use wears like "pièce esquillée ».

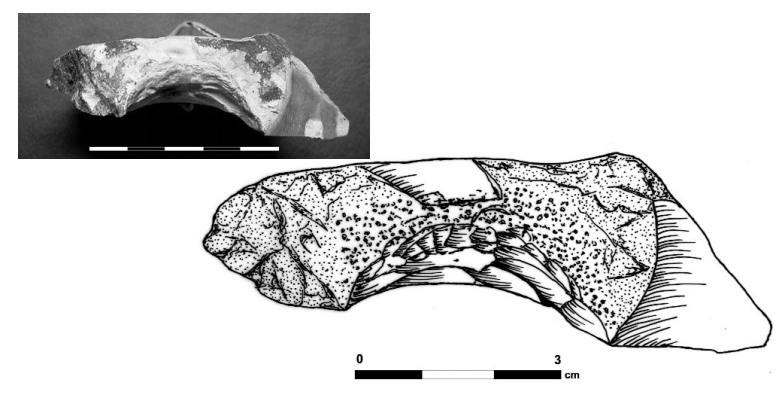

Figure 30 - Éclat de silex utilisé comme coin ; talon percuté formant coche.

Figure 30 - Flake used as a wedge, with notched stroken butt.

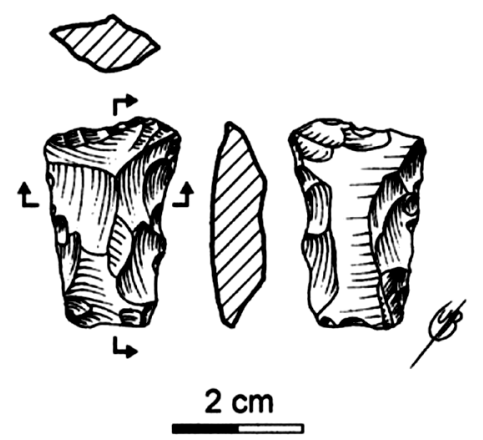

Figure 31 - Pierre à fusil ; vallée du Rio Doce (Brésil). Dessin de M. Brito.

Figure 31 - Flint from a flintlock, Rio Doce Valley. Drawing by $M$. Brito.
Les marques d'impacts sont plus profondes que celles que laisse la percussion libre car le contact est généralement punctiforme, parfois linéaire et vertical - tandis que les percuteurs unipolaires n'ont qu'un contact tangentiel diffus avec la matière travaillée.

Les marteaux en hématite que nous avons utilisés pour enfoncer les coins destinés à fendre du bois ont particulièrement souffert et ont développé une véritable cupule, de contour et de surface irréguliers - ce qui les différencie des quebra cocos destinées à casser les noix de palme. Le percuteur d'aroeira (un bois plus dense que le buis) a également beaucoup souffert de ce travail.

\section{... et l'enclume}

Les enclumes destinées au bris des petites noix de palme indigènes (coquinhos) sont omniprésentes au Brésil. Faites en roches tenaces (diabase, calcaire) ou en galets de quartz faute de mieux, elles peuvent être portables (mesurant alors une dizaine de centimètres) ou peser plusieurs kilogrammes et rester au pied des palmiers. Les chocs répétés provoquent la formation d'une surface déprimée et piquetée fort utile, car elle évite que les noix - lisses et sphériques - ne soient chassées latéralement par un coup mal dirigé. Après un temps variable selon la résistance de la roche, se forme une cupule parfaitement circulaire, de fond finement piqueté, abandonnée quand elle atteint deux centimètres de profondeur (fig. 33). Beaucoup plus rares, les enclumes du Rio Doce probablement destinées au bris d'autres semences dures mais plus grandes et de formes anguleuses (Cansanção) présentent une dépression plus large, irrégulière en plan comme en coupe (notons que nous avons observé des dépressions semblables dans un marché du Haut Atlas marocain, sur des enclumes qui servaient à casser du sucre).

Les pièces archéologiques présentant une surface centrale piquetée sur une ou plusieurs faces ont été systématiquement confondues avec ces casse-noix. Pourtant, une fois reconnue l'existence d'un intense débitage bipolaire, il fallait bien trouver les enclumes qui lui correspondaient. Nous avons alors montré que les enclumes pour le débitage de la pierre présentaient des stigmates bien distincts ; les cicatrices sont généralement linéaires (souvent virgulées), parfois punctiformes ; cela correspond bien aux
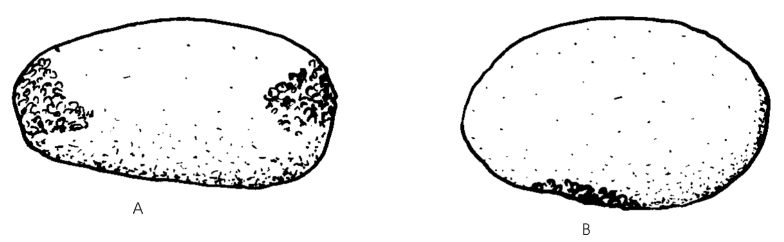

Figure 32 - Localisation des marques d'utilisation sur un percuteur pour taille directe (a) et sur un percuteur pour taille bipolaire (b).

Figure 32 - Use wear on free hand hammerstone (a) and on a bipolar hammerstone (b). 


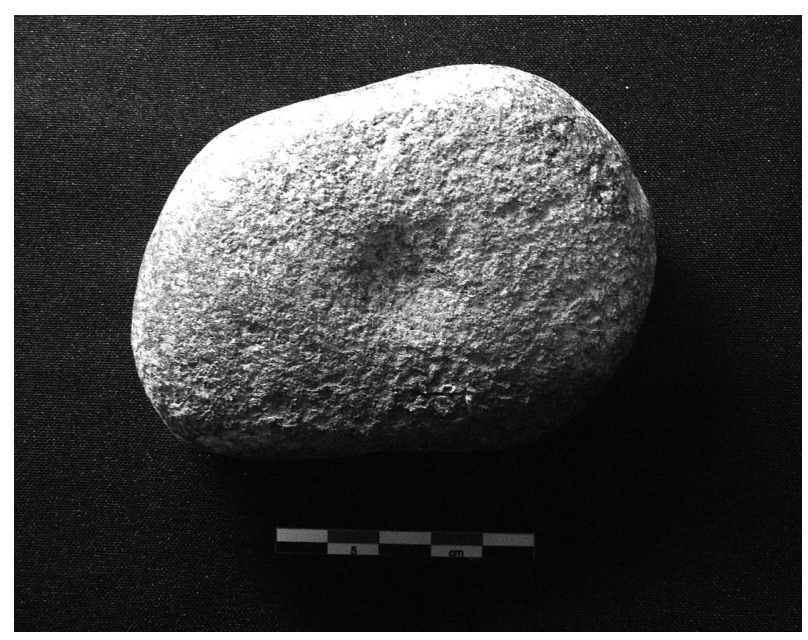

Figure 33 - Enclume préhistorique pour casser les petites noix de palmes américaines.

Figure 33 - Prehistoric anvilstone to crack little american coco nuts.

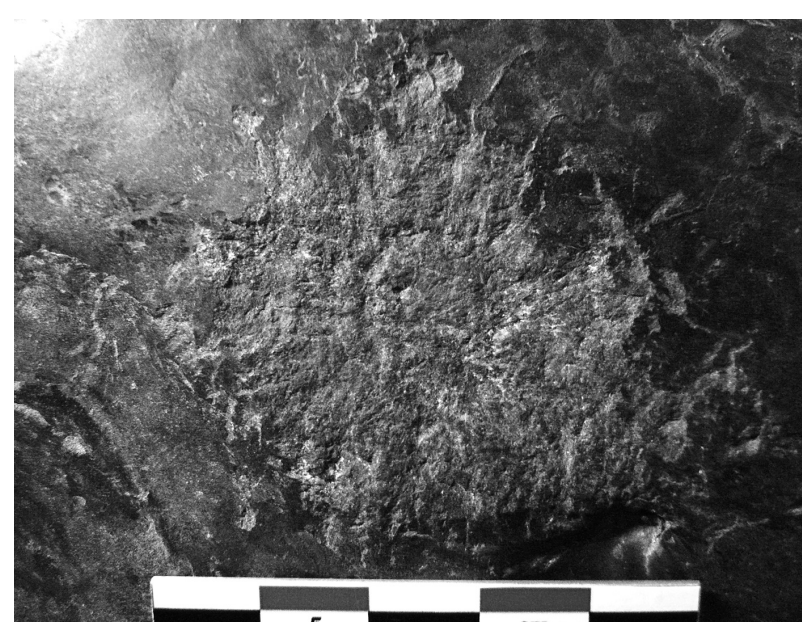

Figure 34 - Enclume expérimentale en quartzite pour tailler le quartz.

Figure 34 - Experimental quartzite anvilstone, used to flake quartz chunks.

lignes (qui, dans le cas du quartz, se forment surtout sur l'enclume) ou aux points d'écrasement (plus souvent formés du côté du percuteur) qui caractérisent plus particulièrement les extrémités percutées des pièces nucléiformes (fig. 34).

Comme les pièces lithiques ne dérapent pas comme les noix sphériques, il n'est pas intéressant de développer une dépression pour les caler ; au contraire, on recherche une surface très dure, alors que les contrecoups successifs provoquent un émiettement qui amortit les chocs. Aussi,

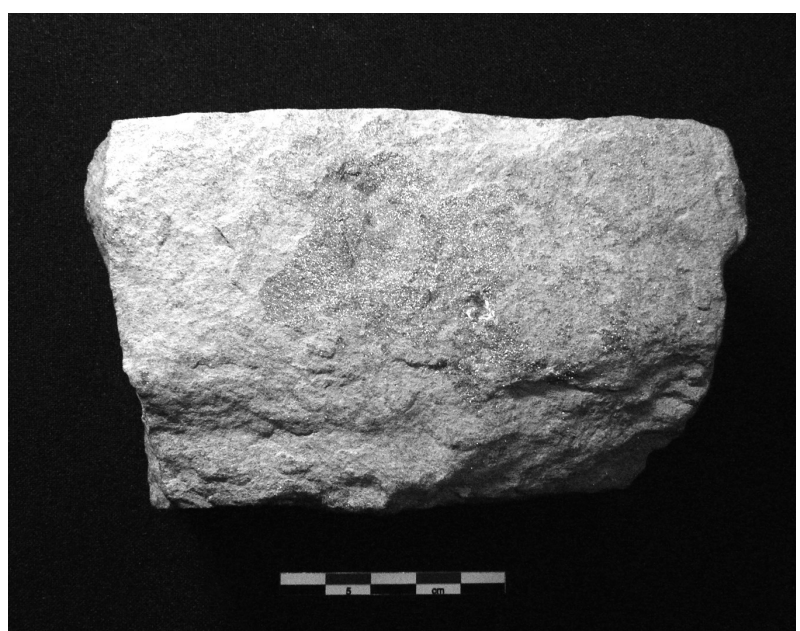

Figure 35 - Enclume expérimentale en hématite compacte, utilisée pour fabriquer des dents de râpe à manioc en diabase et en quartz.

Figure 35 - Experimental hematite anvilstone used to make diabase and quartz teeth for manioc rasps.

les débitages s'étalent-ils sur la surface disponible, au lieu de se focaliser. En conséquence, la surface de travail présente un contour irrégulier et les marques de choc sont moins concentrées.

Notons que certaines roches comme l'hématite compacte réagissent de manière différente et s'écaillent, perdant les marques virgulées, comme le montre la surface d'une des enclumes expérimentales qui nous ont servi à préparer des « dents » de râpe à manioc (fig. 35).

\section{CONCLUSION}

Loin d'être un pis-aller, le débitage sur enclume est bien souvent la méthode la plus efficace si l'on regarde le rendement de tranchant par poids de matière première et la facilité du travail. Elle est moins intéressante quand on désire des supports de morphologie très spéciale et surtout, de grandes dimensions (comme c'est le cas pour certaines industries à lames) et utilisés sans manche. Les industries à lames nous paraissent donc être une exception et non le modèle plus ou moins consciemment recherché - et rarement appliqué dans la plus grande partie du monde - par les tailleurs préhistoriques.

Évidemment, la grande convergence morphologique et la similitude de stigmates rencontrés sur les produits de la taille sur enclume rendent difficile l'identification de « méthodes technologiques » comme celles qui ont permis de différencier les « faciès culturels " sur lesquels se fondent les chronologies européennes, celles du Proche Orient et d'une bonne partie de l'Afrique. Les comparaisons entre les séries archéologiques doivent alors utiliser d'autres éléments que l'industrie lithique taillée, ce qui explique les approches très différentes qu'on observe entre les chercheurs européens et 
ceux de nombreuses autres régions - trop souvent considérées «périphériques » jusqu'à ce jour. La taille sur enclume n'est pas marginale, elle pourrait bien être la mieux représentée dans l'ensemble du monde.

Ruth Benedict et Pierre Clastres réclamaient une « révolution copernicienne » en Anthropologie, par laquelle la vision eurocentrique se trouverait relativisée ; il en faut également une en Préhistoire et la présente Table-Ronde suggère qu'elle est en train de s'effectuer dans l'étude des industries lithiques.

\section{Remerciements}

Nous remercions Solange Caldarelli, qui nous a aimablement autorisé à étudier le matériel inédit provenant des prospections réalisées par Scientia dans l'état du Maranhão et Sérgio da Silveira qui en a fait les photographies ; Rodrigo Lavina, qui nous a confié une collection provenant de ses fouilles dans l'état de Santa Catarina ; E. Fogaça, qui nous a montré des séries de pièces sur galets de l'état de Goiás. Thomas Heyd a révisé l'abstract en anglais. Les planches de dessins de cet article sont de A. Prous ; sauf indication contraire les photographies de $M$. Alonso et G. Neves.

\section{BIBLIOGRAPHIE}

BREUIL H. et LANTIER R. 1951 - Les Hommes de la pierre ancienne - Paléolithique et Mésolithique, Paris, Payot, $335 \mathrm{p}$.

COSTA F. W. 2003 - Análise das indústrias líticas da área de confluência dos Rios Negro e Solimões. São Paulo : Université de São Paulo. Mémoire de Maîtrise, 120 p.

DICKSON F.P. 1977 - Quartz flaking. In : Stone Tools as Cultural Markers : change, evolution and complexity, eds R.Wright, Canberra: Australian Institute of Aboriginal Studies. Prehistory and Material Culture Series $n^{\circ} 13$, pp. 97-103.

DISTEL A. F. 1972/3 - Petroglifos del rio Keros. Anales de Arqueologia y Etnologia, Mendoza, 28, p. 67-78.

FLENNIKEN J. 1981 - A model applied to the vein quartz artifacts of the Hoko River Site Washington State University. Laboratory of Anthropology Reports of Investigations, $\mathrm{n}^{\circ} 59$.

HURT W. 1960 - The cultural complexes from the Lagoa Santa region, Brazil. American Anthropologist, 62 (4), p. $569-585$.

HURT W. 1960 - Te cultural complexes from the lagoa Santa region, Brazil. American Anthropologist, 62 (4), p. 569-585.

HURT W. et BLASI 1969 - O Projeto arqueológico Lagoa Santa. Arquivos do Museu Paranaense, NS, Curitiba, 4, p. 1-63.
JELINEK J. 1976 - Encyclopédie illustrée de l'homme préhistorique, Prague, Gründ, 560 p.

KOBAYASHI H. 1975 - The experimental study of bipolar flakes. In : E. Swanson, (Ed.) Lithic technology : making and using stone tools. World Anthropology / Mouton, The Hague-Paris, p. 115-127.

MAZIERE G. 1984 - La pièce esquillée, outil ou déchet ? Bull. Soc. Préhist. Fr. 81 (6), p. 182-187.

MILLER T. O. 1975 - Tecnologia lítica, arqueologia experimental no Brasil. Anais do Museu da UFSC, Florianóplis, 7 (8), p. 7-93.

MILLER T. O. 1979 - Stonework of the Xetá Indians of Brazil. In : Lithic Use wear Analysis, Academic Press, p. 401-407.

MOURA M. T. et PROUS A. - 1989 Vestígios de utilização em instrumentos líticos utilizados brutos. Dédalo, Univ. São Paulo, Publ. Avuls. 1, p. 409-428.

MOURRE V. 1996 - Les industries de quartz du Paléolithique. Terminologie, méthodologie et technologie. Paléo, 8, p. 205-223.

PETREQUIN P. et PETREQUIN A.-M. 1993 - Écologie d'un outil : la hache de pierre en Irian Jaya (Indonésie), Paris, CNRS, Monographie du CRA 12, 439 p.

PROUS A. 1981 - «Fouilles du grand abri de Santana do Riacho (Minas Gerais, Brésil)" Journal de la Société des Américanistes, Paris, N. S., 67, p. 163-183.

PROUS A. 2004 - Apontamientos para un curso sobre las Indústrias líticas. Fundación Ortegalia, 258 p.

PROUS A. et ALONSO M. 1986 - A tecnologia de debitagem do quartzo no centro de Minas Gerais : lascamento bipolar. Arquivos do Museu de História Natural UFMG, Belo Horizonte, 11, p. 91-111.

PROUS A., ALONSO M., PILO, H., XAVIER L., LIMA A. et SOUZA G. 2002 - Os machados pré-históricos no Brasil : descrição de coleções brasileiras e trabalhos experimentais : fabricação de lâminas, cabos, encabamento e utilização. Canindé, Universidade Federal de Sergipe, 2, p. 161-236.

PROUS A. et LIMA M.-A. 1990 - "A tecnologia de debitagem do quartzo no Centro de Minas Gerais", Arquivos do Museu de História natural, Vol. XI, 1986/1990, pp. 91-114.

RAVERE A. 1975 - «Tool Making and Tool Use Among the Preceramic People of Panama» in Swanson, E. ed. Lithic Technology - Making and using stone tools, World Anthropology/Mouton, The Hague, Paris, p. 173-209.

REICHEL-DOLMATOFF G. 1997 - Chamanes de la selva pluvial - Ensayos sobre los Indios Tukano del Noroeste amazonico, Themis Books, Dartington, 344 p. 
ROSTAIN S. 1994 - L'occupation amérindienne ancienne du littoral de Guyane, Paris, Univ. de Paris 1, Thèse, tome 1, $536 \mathrm{p}$.

SCHMITZ P. I., ARTUSI L., JACOBUS A., ROGGE J., MARTIN H. et BAUMHARDT G. 1990 - Uma aldéia Tupiguarani : Projeto Candelária, RS Documentos $\mathrm{n}^{\circ} 4$, Instituto Anchietano de pesquisas, São Leopoldo, 135 p.
RUGENDAS J. M. - (original 1835) 1989 - Viagem pitoresca através do Brasil, Editora da USP, São Paulo (traduction de l'original publié em français et em allemand à Paris em 1835), $166 \mathrm{p}$.

SCHMITZ P. I., ROGGE J., OSÓRIO ROSA A. et BEBER M. V. 1998 - Aterros indígenas no Pantanal do Mato Grosso do Sul. Pesquisas, Antropologia, S. Leopolodo, 54, 271 p. Van Riet Lowe, C. 1952 - The Pleistocene geology and Prehistory of Uganda, t. 6, 113 p. 


\section{Tableau des formes « classiques » obtenues par débitage bipolaire sur quartz}

\section{Techno-typologie des produits de débitage du quartz}

Les caractéristiques du quartz amènent à considérer un nombre très élevé de débris - éventuellement utilisables et ne pouvant donc pas être exclus des analyses. Nous avons donc établi une nomenclature pour les produits de débitage bipolaire (qui ne s'applique pas exclusivement au quartz, cf. Prous et Lima 1990).

Au Brésil, nous utilisons les catégories suivantes, qui ont l'avantage de distinguer nettement les produits de taille unipolaire de ceux de la taille sur enclume :

\section{Identification technologique :}

1 - Débitage « bipolaire » (= sur enclume) :

- Pièces nucléiformes (et fragments de pièces nucléiformes)

ortholites (sur galet)

biconiques,

prismatiques

rectangulaires (pièces esquillées)

- Éclats bipolaires (et fragments d'éclats bipolaires)

Éclats ovales initiaux de galet

Lamelles bipolaires

Éclats ultra fins et plats

- Cassons Autres éclats bipolaires

Aiguilles, baguettes

Débris polyédriques de concassage

- Autres

Esquilles

- Poudre

2 - Débitage unipolaire :

- Nucléus

Monocristaux

Galets

Blocs polyédriques

- Éclats de divers types

- Cassons

- Esquilles

- Poudre

\section{3 - Technique mixte :}

Attaque unipolaire et reprise bipolaire (de prisme ou de galet)

Attaque bipolaire et reprise unipolaire (de galet)

Alternance unipolaire/bipolaire.

4 - Pièces d'origine technologique douteuse :

Éclats et fragments qui pourraient avoir été débités aussi bien sur enclume qu'à main libre.

La description et classification typologique des pièces retouchées suit les règles traditionnellement utilisées pour les supports de silex, de quartzite ou d'obsidienne. 
